\title{
Dynamics of gravity-driven viscoelastic films on wavy walls
}

\author{
Arjun Sharma \\ Department of Aeronautics, Imperial College London, London SW7 2AZ, UK \\ Prasun K. Ray and Demetrios T. Papageorgiou \\ Department of Mathematics, Imperial College London, London SW7 2AZ, UK
}

\begin{abstract}
The linear stability and nonlinear dynamics of viscoelastic liquid films flowing down inclined surfaces with sinusoidal topography are investigated. The Oldroyd-B constitutive model is used, and numerical solutions of a long-wave nonlinear evolution equation for the film thickness (introduced by Davalos-Orozco[1]) provide insight into the influence of elasticity and wall topography on the nonlinear film dynamics, while Floquet analysis of the linearized evolution equation is used to study the onset of linear instability. Focusing initially on inertialess films (with zero Reynolds number), linear stability results are organized into three regimes based on the wall wavelength. For sufficiently short and sufficiently long wall wavelengths, the onset of instability is not tangibly affected by the topography. There is, however, an intermediate range of wavelengths where, as the wall wavelength is increased, the critical Deborah number for the onset of instability first decreases (topography is destabilizing) and then increases sufficiently for topography to be stabilizing (relative to the flat wall). Solutions to a perturbation amplitude equation indicate that the character of the instability changes substantially within this intermediate range; topography induces streamwise variations in the base-state velocity at the free surface which couple with perturbations and substantially influence the instability growth rate. Very similar trends are observed for Newtonian films and variations in the critical Reynolds number. Simulations of the full nonlinear evolution equation produce a broad range of nonlinear states including traveling waves, time-periodic waves, and chaos. Perturbations to the film generally saturate at higher amplitudes for cases with larger linear growth rates (e.g. with increasing Deborah number or for a destabilizing wall wavelength), and topography introduces finer temporal scales in the dynamics. The qualitative influences of inclination and inertia on the nonlinear dynamics are shown to be simply related to the influence of elasticity using analytical linear stability results for the flat-wall case.
\end{abstract}




\section{INTRODUCTION}

Gravity-driven liquid film flows are relevant to a number of important industrial applications and coating processes. These processes generally require careful control of the film thickness, process speed, and final material properties[2]. Accordingly, the dynamics of films on flat surfaces have been extensively studied. Early investigators found that gravity-driven Newtonian films become linearly unstable if the Reynolds number is sufficiently large[3], and the subsequent development of nonlinear waves from this inertial instability has also been a topic of substantial interest (see the monograph by [4] and the reviews by [5] and [6]). There has been a specific focus on the dynamics of long nonlinear waves in flows with large surface tension where the governing equations can be substantially simplified using the Benney, integral boundary-layer (IBL), and weighted-residual approximations.

In many practical applications, the film is viscoelastic, and early studies investigated the linear stability of viscoelastic gravity-driven films using second-order fluid models for the stress[7-9]. These studies found that elasticity enhanced inertial instability, however second-order models assume that a representative fluid relaxation time is small relative to a characteristic time scale of the flow (i.e. a Deborah number, De, should satisfy $D e \ll 1$ ) and are best-suited for steady or quasi-steady ('slow') flows[10]. The Oldroyd-B constitutive model is better-suited for unsteady shear flows with substantial viscoelastic effects, and subsequent linear stability studies using this model (or the closely-related Upper-convected Maxwell model) confirmed the destabilizing influence of viscoelasticity and also revealed that an elastic instability may be present if the Deborah number is sufficiently large (even when the Reynolds number is zero)[11-15]. Viscoelastic analogues to the Benney[16-18] and IBL[19] equations have also been derived and analyzed, and numerical simulations of both the Newtonian and viscoelastic Benney equations indicate that a variety of nonlinear states including travelling waves and chaos may be excited (see [20] and section V). Dandapat \& Gupta[16] and Joo[17] utilized second-order fluid and 4-constant Oldroyd constitutive models, respectively (the latter produces weak shear-thinning unlike the second-order and Oldroyd-B models), and focused on the modification of nonlinear inertial waves by viscoelasticity. Kang \& Chen[18] noted that viscoelastic effects are likely to be dominant in very thin films and focused on the dynamics associated with elastic instability. They used the 
Oldroyd-B model, carried out an asymptotic expansion to 2nd order, and then investigated the weakly-nonlinear dynamics generated by their evolution equation and the corresponding bifurcation structure. Here, we also use the Oldroyd-B model and focus on elastic instability (results with finite Re are also included) though we truncate our expansion at 1st order. The Oldroyd-B model has been widely used in studies of shear flows of dilute polymeric solutions and can be derived from a kinetic theory in which the polymer molecules are modeled as noninteracting Hookean elastic dumbbells[21]. It is a simple model that reasonably describes significant viscoelastic effects in shear flows of dilute highly-elastic liquids at moderate shear rates (e.g. memory effects and a finite streamwise normal stress). At higher shear rates, a few important weaknesses emerge. The Oldroyd-B model produces a shear-independent viscosity and a constant first normal-stress coefficient and does not capture the shear-thinning behavior exhibited by most fluids of interest. Other general weaknesses include the development of unphysically large tensile stresses in extensional flows (since linear Hookean springs can stretch indefinitely), zero transverse normal stress components in simple shear flow, and the assumption of a single representative fluid relaxation time. Despite these shortcomings, the model's relative simplicity and analytical tractability make it well-suited for theoretical studies of elastic instabilities in viscoelastic shear flows with small or moderate shear rates $[22]$.

Protective coatings are often required on devices with uneven surfaces, and even nominally flat walls often have surface imperfections which introduce perturbations into films and influence the quality of the final polymer coating[23]. Thus it is important to consider film flow on walls with topography, and in this work, we investigate the stability and dynamics of gravity-driven viscoelastic films on sinusoidally-varying surfaces (figure 1). A number of studies have investigated the influence of sinusoidal topography on film stability and dynamics for Newtonian films[e.g. 24-29] while power-law[30] and weakly-viscoelastic (Walters-B)[31] films have also been considered. The power-law model produces a sheardependent viscosity which is relevant for concentrated polymeric solutions and melts which are both typically strongly shear-thinning. The power-law model does not, however, include memory effects or generate normal stresses in simple shear flows. In contrast, Oldroyd-B fluids do exhibit these important viscoelastic effects but do not exhibit shear thinning (or thickening). The Walters-B model is an example of a second-order model and as noted above, 
is best-suited for quasi-steady flows where viscoelastic effects are weak. Taken together, these studies of Newtonian and Non-Newtonian films utilize a variety of approaches and a broad range of parameters, and stabilization due to topography was observed by [25, 28, 30], while [29] and [26] found that destabilization could also occur for some wall wavelengths. Of particular relevance to the present work is Dávalos-Orozco[1] where an Oldroyd-B fluid was considered, and the viscoelastic Benney equation (VBE) introduced by [18] was modified to account for the wall deformation. Numerical simulations of this nonlinear evolution equation with spatially-localized time-harmonic forcing were used to show that topography could be stabilizing for appropriately chosen wall deformations. Here, we use this modified VBE as a starting point and: 1) use Floquet analysis to systematically analyze its linear stability while also exploring the detailed elastic instability mechanism and 2) use extensive numerical simulations of the full evolution equation to investigate the nonlinear states which develop from the linear instability. These new linear stability results provide a clear picture of the influence of sinusoidal wall topography on long-wave elastic instability and provide a framework for designing optimal wall deformations for a given application. Along similar lines, the nonlinear simulation results provide qualitative guidance for interpreting laboratory observations of unsteady viscoelastic film flows over topography. This paper is organized as follows. In section II we discuss the mathematical formulation of the nonlinear partial differential equation governing the evolution of the film thickness for viscoelastic film flow on walls with topography. In section III, we show the construction of the steady state both numerically and analytically (for the case where the wall deformation amplitude is small) and then present linear stability results obtained using Floquet analysis in section IV. Finally, in section $\mathrm{V}$ we use numerical simulations of the full nonlinear evolution equation to explore the unsteady dynamics of this flow.

\section{FORMULATION}

In this section, we outline the derivation of the modified VBE introduced in [1]. A few intermediate steps are omitted as the basic approach is very similar to the derivation of the well-known Benney equation[32]. We consider the evolution of long waves on a film with

thickness $h\left(x_{1}, t\right)$ overlying a wall with surface, $s\left(x_{1}, t\right)$, inclined at an angle $\theta$ as shown 


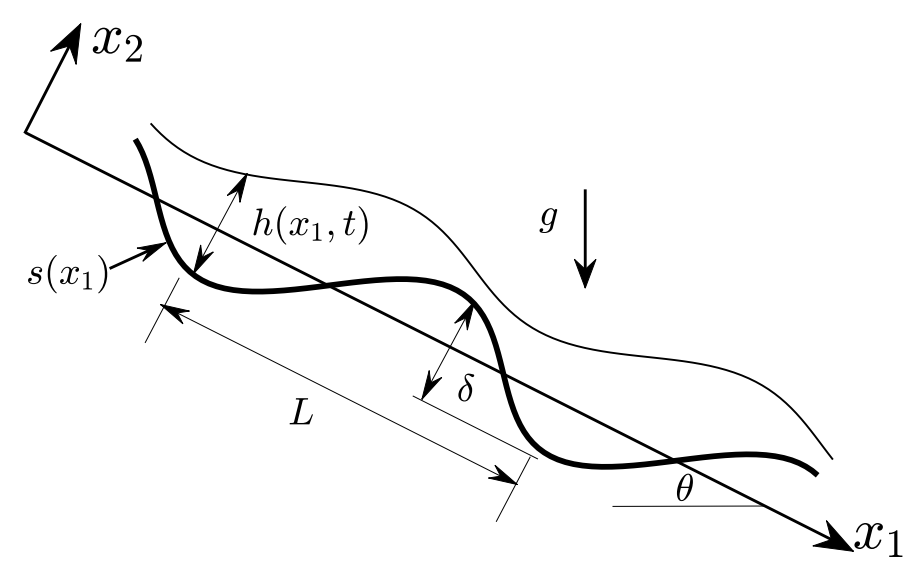

FIG. 1: Schematic of Oldroyd-B liquid film flowing down an inclined topography

in figure 1. We start from the dimensionless Navier-Stokes equations for two-dimensional incompressible flow modified with a polymer stress term to account for the influence of viscoelasticity,

$$
\begin{gathered}
\frac{\partial u_{j}}{\partial x_{j}}=0 \\
R e\left(\frac{\partial u_{i}}{\partial t}+u_{j} \frac{\partial u_{i}}{\partial x_{j}}\right)+\frac{\partial p}{\partial x_{i}}=F_{i}+\beta \frac{\partial^{2} u_{i}}{\partial x_{j} \partial x_{j}}+(1-\beta) \frac{\partial a_{j i}}{\partial x_{j}},
\end{gathered}
$$

where $u_{i}$ is velocity, $p$ is pressure, $(1-\beta) a_{i j} / R e$ is the polymer stress, $F_{i}$ represents the force due to gravity with $\left(F_{1}, F_{2}\right)=\left(3,-3 C_{t}\right)$, and the density, $\rho$, is uniform and constant. Additionally, $R e=u_{N} h_{N} / \nu$ is the Reynolds number, $\beta=\nu_{s} / \nu$ is the ratio of the solvent and solution viscosities, and $C_{t}=\cot \theta$ is an inclination parameter. These parameters have been formed using the Nusselt flat film thickness, $h_{N}$, and bulk velocity, $u_{N}$, which is related to gravity, $g$, and kinematic viscosity, $\nu$, through, $u_{N}=g \sin \theta h_{N}^{2} /(3 \nu)$. The polymer stress is obtained from the Oldroyd-B constitutive model,

$$
\frac{\partial a_{i j}}{\partial t}+u_{k} \frac{\partial a_{i j}}{\partial x_{k}}-a_{i k} \frac{\partial u_{j}}{\partial x_{k}}-a_{k j} \frac{\partial u_{i}}{\partial x_{k}}=\frac{1}{\mathrm{De}}\left(\frac{\partial u_{i}}{\partial x_{j}}+\frac{\partial u_{j}}{\partial x_{i}}-a_{i j}\right),
$$

where $D e=\lambda u_{N} / h_{N}$ is the Deborah number, $\lambda$ is a characteristic polymer relaxation time, and the Deborah number will be restricted to be $O(1)$. This constraint leads to simplifications to the upper-convected derivative in (2) which are necessary for analytical progress. As 
noted in the introduction, the Oldroyd-B model has a few well-known limitations (e.g. [33]) which are more pronounced at larger values of $D e$, and with $D e \sim O(1)$, we expect the model to capture important qualitative trends associated with streamwise normal stresses and memory effects. We require $u_{i}=0$ at the wall and enforce continuity of tangential and normal stress at the free surface (see the appendix). Using the no-slip condition and the continuity equation, the kinematic condition for the free surface can be written as,

$$
\frac{\partial h}{\partial t}+\frac{\partial q}{\partial x_{1}}=0, \quad q=\int_{s}^{h+s} u_{1} \mathrm{~d} x_{2}
$$

The normal stress condition introduces a surface tension parameter (an inverse Bond number), $S=\gamma /\left(\mu u_{N}\right)$. This parameter is assumed to be large, $S \sim O\left(1 / \epsilon^{2}\right)$, and we also assume that the wall deformation varies slowly such that

$$
h_{\text {typical }} / L=\epsilon \ll 1
$$

where $h_{\text {typical }}$ is a 'typical' film thickness and $L$ is the deformation wavelength. These assumptions lead to a focus on waves varying slowly in both the streamwise direction and time. Introducing the slow variables $\tilde{t}=\epsilon t$ and $\tilde{x}_{1}=\epsilon x_{1}$, the dependent variables are expanded in powers of $\epsilon$ (see the appendix). Substituting this expansion into the governing equations and boundary conditions, and solving the resulting equations at $O(1)$ and $O(\epsilon)$, the kinematic condition (up to $O\left(\epsilon^{2}\right)$ ) can be written as,

$$
\frac{\partial h}{\partial t}+\frac{\partial}{\partial x_{1}}\left[h^{3}+\frac{6}{5} \operatorname{Reh}^{6} \frac{\partial h}{\partial x_{1}}-C_{t} h^{3} \frac{\partial(h+s)}{\partial x_{1}}+\frac{1}{3} S h^{3} \frac{\partial^{3}(h+s)}{\partial x_{1}^{3}}+3 \mathcal{D} h^{4} \frac{\partial h}{\partial x_{1}}\right]=0,
$$

where we have reverted back to the physical variables, $x_{1}$ and $t$. Further details on this derivation are provided in the appendix. The first term within the brackets in equation (5) arises due to the shear generated by the combination of the no-slip condition and gravitational forcing along the $x_{1}$ direction; the second to fourth terms represent the effects of inertia, inclination, and surface tension, respectively, and the fifth term represents the influence of elasticity with $\mathcal{D} \equiv D e(1-\gamma)$. This equation matches the evolution equation introduced in [1] (after accounting for differences in non-dimensionalization), and for the case of a flat wall with $s=0$ and $R e=0$, we recover the VBE derived by [18] up to $O(\epsilon)$ (and again after accounting for their non-dimensionalization). The derivation of equation (5) assumes that $C_{t}, R e \ll 1 / \epsilon$; for Newtonian fluids, the numerical solutions of this equation 
have been shown to be very close to numerical solutions of the full Navier-Stokes equations up to $R e \approx 5[20,34]$.

Equation (5) is valid for general slowly-varying topography, however for simplicity, we will only consider sinusoidal wall profiles with wavelength, $L$, and amplitude, $\delta / 2$ :

$$
s\left(x_{1}\right)=\frac{1}{2} \delta \cos \left(\kappa x_{1}\right), \kappa=2 \pi / L
$$

The 'steepness', $\delta / L$, is an important parameter in the discussion below and is required to be $O(\epsilon)$ or smaller.

\section{Polymer stress}

We can infer that the influence of viscoelasticity is destabilizing from equation (5) (see section IV), and a fuller view of this influence and the importance of the streamwise normal stress can be obtained by examining the coupling between the polymer stress components and the film thickness. Elasticity enters equation (5) via the $O(\epsilon) x_{1}$-momentum equation, and using the Oldroyd-B equation we find,

$$
\begin{gathered}
\frac{\partial a_{11}^{(0)}}{\partial \tilde{x}_{1}}=-36 D e\left(\left(\frac{\partial h}{\partial \tilde{x}_{1}}+\frac{\partial s}{\partial \tilde{x}_{1}}\right)\left(x_{2}-s\right)-\frac{\partial s}{\partial \tilde{x}_{1}} h\right)+36 D e \frac{\partial h}{\partial \tilde{x}_{1}} h \\
\frac{\partial a_{12}^{(1)}}{\partial x_{2}}=36 D e\left(\left(\frac{\partial h}{\partial \tilde{x}_{1}}+\frac{\partial s}{\partial \tilde{x}_{1}}\right)\left(x_{2}-s\right)-\frac{\partial s}{\partial \tilde{x}_{1}} h\right)-27 D e \frac{\partial h}{\partial \tilde{x}_{1}} h
\end{gathered}
$$

It is the sum of these terms which appears in the momentum equation, and after substantial cancellations, we arrive at a fairly simple result which is $x_{2}$-independent and strongly dependent on streamwise variations in the film thickness:

$$
\frac{\partial a_{11}^{(0)}}{\partial \tilde{x}_{1}}+\frac{\partial a_{12}^{(1)}}{\partial x_{2}}=\frac{9}{2} D e \frac{\partial\left(h^{2}\right)}{\partial \tilde{x}_{1}}
$$

This result represents competition between the streamwise-normal and shear components of the polymer stress. Crucially, the magnitude of the normal-stress is larger, and overall, elasticity 'pushes' fluid in the streamwise direction towards local maxima in the film thickness and away from local minima. This then is clearly a destabilizing effect and is very similar to the description of the long-wave linear elastic instability mechanism in gravity-driven 
films on flat walls presented in [15]. Equations 7 and 8 also indicate that the wall topography, $s$, directly influences the individual polymer stress components but it is only via the film thickness, $h$, that the topography affects the overall influence of elasticity on the film dynamics.

\section{STEADY STATE}

Steady-state film profiles provide important insight into the response of the film flow to wall topography and are also needed for linear stability calculations. The computation of these profiles is considerably more complicated than the flat-wall case where we simply have $h_{0}=1$. Setting the flux to $q=q_{0}=1$, the governing equation is,

$$
h_{0}^{3}+\frac{6}{5} \operatorname{Reh}_{0}^{6} \frac{d h_{0}}{d x_{1}}+3 \mathcal{D} h_{0}^{4} \frac{d h_{0}}{d x_{1}}-C_{t} h_{0}^{3} \frac{d}{d x_{1}}\left(h_{0}+s\right)+\frac{1}{3} S h_{0}^{3} \frac{d^{3}}{d x_{1}^{3}}\left(h_{0}+s\right)=q_{0}=1,
$$

and we will only consider periodic solutions. Generally, this equation must be solved numerically, and following the formulation of Tseluiko et al.[29], steady states are computed after first writing eq. (9) as a system of first-order non-autonomous ODEs on the domain [0, L] with $f_{1}=h_{0}, f_{2}=d h_{0} / d x_{1}, f_{3}=d^{2} h_{0} / d x_{1}^{2}$ and,

$\frac{d f_{1}}{d x_{1}}=f_{2}, \quad \frac{d f_{2}}{d x_{1}}=f_{3}, \quad \frac{d f_{3}}{d x_{1}}=\frac{3}{S f_{1}^{3}}\left(1-f_{1}^{3}-\left(\frac{6}{5} R e f_{1}^{6}+3 \mathcal{D} f_{1}^{4}\right) f_{2}+C_{t} f_{1}^{3}\left(f_{2}+\frac{d s}{d x_{1}}\right)\right)-\frac{d^{3} s}{d x_{1}^{3}}$.

Periodic boundary conditions are implemented as, $f_{i}(0)=f_{i}(L), i=1,2,3$, and equation (10) is solved using the Matlab suite bvp4c[35] which is an iterative adaptive-mesh boundary-value-problem solver that uses finite-difference discretizations. We only consider steady states with fixed volume flow rates $\left(q_{0}=1\right)$, however a reasonable alternative is to fix the average film height (or equivalently the total volume of fluid). The former case is typically used in experiments while the latter has been frequently used in stability studies and nonlinear simulations of films on topography[36]. Tseluiko et al.[29] considered both approaches in their study of inertial instabilities on films over topography, and found qualitatively similar results for the critical Reynolds number. Preliminary calculations for viscoelastic films with fixed fluid volumes (not shown here) show qualitatively similar results for the critical Deborah number, however further investigation of the fixed-volume case would be a useful complement to the present study. 


\section{A. Asymptotic Solution}

The results presented below largely correspond to cases where the amplitude of the wall deformation is small. In such cases, an approximate analytical solution for the steady-state film thickness can be found using asymptotic expansions. The steady state surface elevation $F\left(x_{1}\right)=h_{0}\left(x_{1}\right)+s\left(x_{1}\right)$ is expanded as,

$$
F\left(x_{1}\right)=1+\delta F_{1}\left(x_{1}\right)+\mathcal{O}\left(\delta^{2}\right)
$$

and after substituting this expansion into equation (10), $F_{1}$ can be found in a straightforward manner using Fourier transforms (see [37]):

$F_{1}\left(x_{1}\right)=A_{1} \cos \left(\kappa x_{1}-\phi_{1}\right), \quad A_{1}=\frac{1}{2} \sqrt{\frac{9+\kappa^{2} V^{2}}{9+\kappa^{2}\left(V-\mathcal{P}_{\kappa}\right)^{2}}}, \quad \phi_{1}=-\operatorname{atan}\left(\frac{3 \kappa \mathcal{P}_{\kappa}}{9+\kappa^{2} V\left(V-\mathcal{P}_{\kappa}\right)}\right)$.

The influences of elasticity and inertia are condensed into a single parameter, $V$, while $\mathcal{P}_{\kappa}$ contains the effects of surface tension and inclination,

$$
V=\frac{6}{5} R e+3 \mathcal{D}, \quad \mathcal{P}_{\kappa}=C_{t}+\frac{1}{3} \kappa^{2} S .
$$

The bulk of the results presented below correspond to cases where $\mathcal{P}_{\kappa}>V$, and with our chosen parameter values, numerical calculations indicate that $\mathcal{P}_{\kappa}$ typically controls the profile shapes. Taking the extreme case, $V=0$, we see that the amplitude of free-surface oscillations, $A_{1}$, decreases as any one of $\kappa, S$, and $C_{t}$ are increased. The selective damping of higher-wavenumber oscillations by surface tension is expected, and here we see that inclination has a similar effect though the relative importance of the inclination angle decreases as the topography wavenumber increases.

Steady state results for inertialess films with $\mathcal{D}=0.2$ and $C_{t}=1$ are shown in figure 2. With a fixed wall amplitude, $\delta=0.2$, the free surface is relatively flat for the smaller wall wavelengths and tends to synchronize with the wall at larger wavelengths (figure 2a). The influence of the amplitude, $\delta$, can be accounted for by normalizing the free surface height with, $F_{\text {norm }}=\left(F\left(x_{1}\right)-1\right) / \delta+1$. From the asymptotic results above, we expect this normalized height to be independent of the wall amplitude if $L$ and $S$ are fixed, and numerical results shown in figure $2 \mathrm{~b}$ confirm this expectation. In the next section, it will be useful to compare results with the steepness, $\delta / L$, held fixed. Steady state results for $\delta / L=0.2$ are 

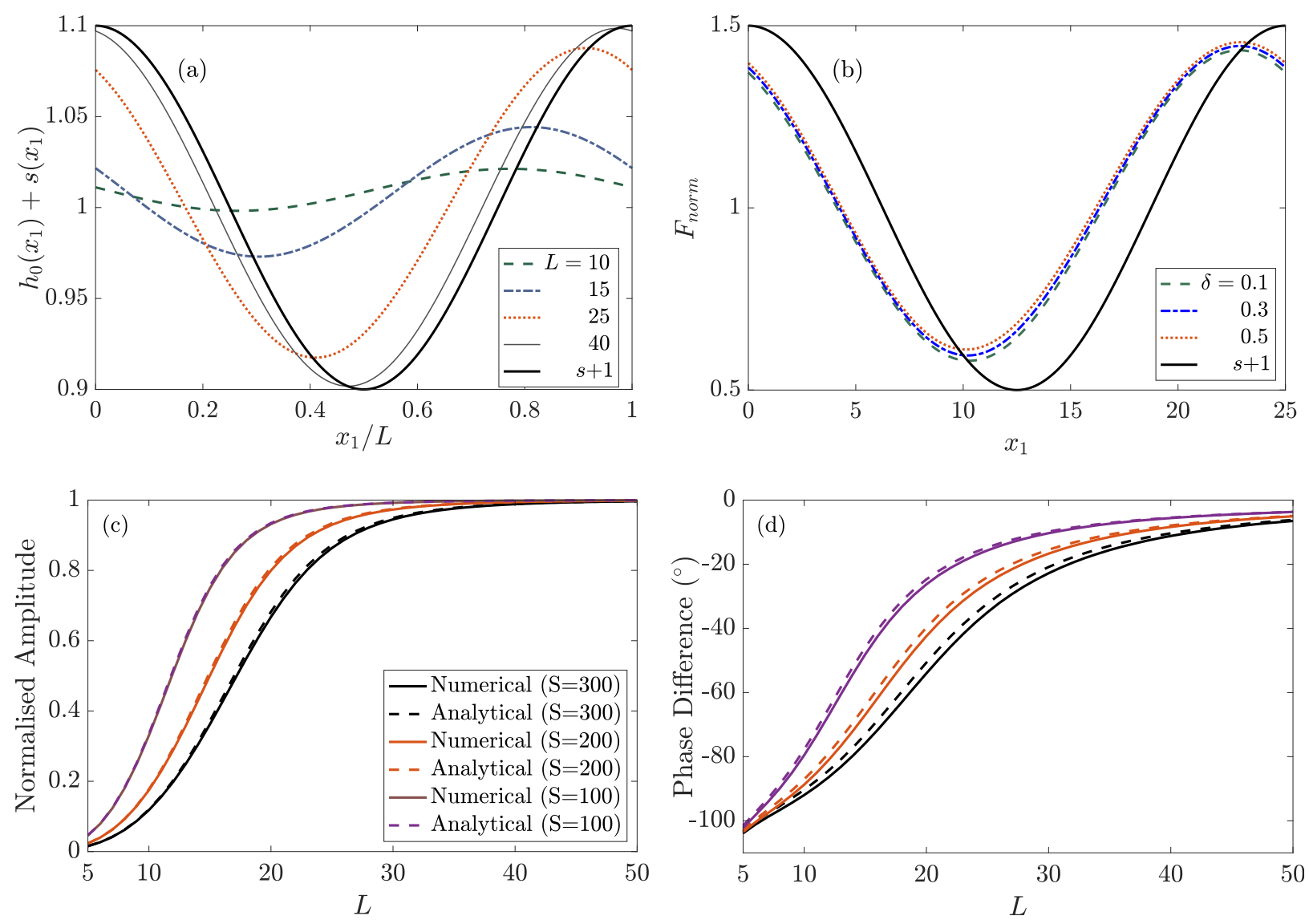

FIG. 2: Influence of sinusoidal wall on the steady state for $R e=0, \mathcal{D}=0.2$ and $C_{t}=1$. Numerical Solutions $(S=300)$ : (a) Interface profiles for fixed wall amplitude, $\delta=0.2$; (b)

Normalised interfacial profiles, $F_{\text {norm }}$ for fixed wall wavelength, $L=25$. Numerical and Analytical Solutions $(S=100,200$ and 300) with fixed wall steepness, $\delta / L=0.02$, and varying wall wavelength, L: (c) Amplitude of $F_{n o r m}$; (d) phase difference between the interface and the wall (same legend as (c)).

summarized in figures $2 \mathrm{c}$ and $2 \mathrm{~d}$. Asymptotic solutions are also included here, and there is good agreement between analytical results and numerical solutions to the full equations (9). For relatively small wall wavelengths, the free surface flattens due (primarily) to surface tension, and at large wavelengths the film thickness tends towards a constant, $x_{1}$-independent value. At intermediate wavelengths, both the normalized amplitude and the phase difference change substantially as the film transitions from a flat free surface to a sinusoidal profile which is in-phase with the wall. Reducing the surface tension produces a shift towards shorter wavelengths. Below, we consider flows with $0 \leq \mathcal{D} \lesssim 1$ and $0 \leq R e \lesssim 2.5$ and the 
trends shown in figure 2 are observed throughout this parameter space.

\section{LINEAR STABILITY}

We now examine the influence of sinusoidal topography on the linear stability of computed steady film profiles. After presenting the problem formulation, we move on to numerical results with a focus on inertialess flows $(R e=0)$. For flat walls, elastic instability is present when $\mathcal{D}>1 / 3 C_{t}$ (see below), and the question then is, how does topography with a given wavelength, $L$, and amplitude, $\delta$, modify this critical elasticity? We will principally consider wavelengths in the range, $5 \leq L \lesssim 60$, and amplitudes, $\delta \lesssim 1$. The surface tension will be set to $S=100,200$, or 300 , and these choices ensure both that the wall topography exerts a non-trivial influence on the flow stability and that the modelling assumptions required for equation (5) are satisfied. The physical mechanisms underlying the topography-induced

changes to $\mathcal{D}_{\text {crit }}$ are also discussed in detail. This discussion centers on a disturbance energy equation obtained from our linearized kinematic condition which allows us to isolate the influence of topography on the elastic instability.

\section{A. Formulation}

The formulation of the linear problem begins with the addition of a small perturbation to the steady state,

$$
h\left(x_{1}, t\right)=h_{0}\left(x_{1}\right)+\eta\left(x_{1}, t\right) .
$$

Substituting this expression into equation (5) and linearizing gives,

$$
\frac{\partial \eta}{\partial t}=\mathcal{L}[\eta]=-\frac{\partial}{\partial x_{1}}\left(b_{0} \eta+b_{1} \frac{\partial \eta}{\partial x_{1}}+b_{2} \frac{\partial^{3} \eta}{\partial x_{1}^{3}}\right)
$$

where $b_{0}\left(x_{1}\right), b_{1}\left(x_{1}\right)$ and $b_{2}\left(x_{1}\right)$ are periodic functions of the steady state and wall topography:

$$
\begin{aligned}
& b_{0}\left(x_{1}\right)=3 h_{0}^{2}+\left(\frac{36}{5} \operatorname{Reh}_{0}^{5}+12 \mathcal{D} h_{0}^{3}\right) \frac{\partial h_{0}}{\partial x_{1}}-3 C_{t} h_{0}^{2} \frac{\partial\left(h_{0}+s\right)}{\partial x_{1}}+S h_{0}^{2} \frac{\partial^{3}\left(h_{0}+s\right)}{\partial x_{1}^{3}}, \\
& b_{1}\left(x_{1}\right)=\frac{6}{5} R e h_{0}^{6}+3 \mathcal{D} h_{0}^{4}-C_{t} h_{0}^{3}, \quad b_{2}\left(x_{1}\right)=\frac{1}{3} S h_{0}^{3} .
\end{aligned}
$$

For a flat wall $(s=0)$, the steady state is $h_{0}=1$, and linear stability analysis is straightforward. Introducing normal modes, $\eta\left(x_{1}, t\right)=\hat{\eta} \exp \left(i\left(k x_{1}-\omega t\right)\right)+$ c.c., the growth rate is, 


$$
\omega_{i}=k^{2}\left(\frac{6}{5} R e+3 \mathcal{D}-C_{t}-\frac{1}{3} k^{2} S\right)
$$

where $\omega=\omega_{r}+i \omega_{i}$. The phase speed is, $c_{p h}=\omega_{r} / k=3$, and instability occurs when,

$$
V=\frac{6}{5} R e+3 \mathcal{D}>C_{t}+\frac{1}{3} k^{2} S
$$

We see that elasticity and inertia are destabilising whereas surface tension and reduction of inclination (increase of $C_{t}$ ) are stabilising. Equivalent expressions for the growth rate in inertialess flow were given by [15] and [18] with the former also neglecting surface tension. These results apply to long waves, and [13] presented results for instabilities with $O(1)$ wavenumbers.

With a sinusoidal wall, we can analyze the stability problem by utilizing Floquet theory and assuming perturbations take the form,

$$
\eta\left(x_{1}, t\right)=\exp \left(\sigma t+i k x_{1}\right) g\left(x_{1}\right)+c . c .,
$$

where $k \in[-\pi / L, \pi / L]$, and $g\left(x_{1}\right)$ is $L$-periodic. Substituting equation (19) into (15) gives an eigenvalue problem,

$$
\mathcal{L}_{k}[g]=\sigma g
$$

where $\mathcal{L}_{k}[g]=\exp \left(-i k x_{1}\right) \mathcal{L}\left[\exp \left(i k x_{1}\right) g\right]$, and each $\mathcal{L}_{k}$ acts over the finite domain of size $L$. The eigenvalue spectrum of $\mathcal{L}, \Sigma(\mathcal{L})$, is the union over all $k$,

$$
\Sigma(\mathcal{L})=\bigcup_{k \in[-\pi /(L), \pi /(L)]} \Sigma\left(\mathcal{L}_{k}\right)
$$

After solving for the base flow, a series of eigenvalue problems corresponding to different values of $k$ are solved in Matlab. Specifically, we vary $k$ in the range $[-\pi / L, \pi / L]$ with increments of $\pi /(50 L)$. Cases with $k=0$ and $k=\pi / L$ correspond to the fundamental and sub-harmonic modes, respectively, however we find that other 'detuned' modes are typically the most unstable (see figure 5b). Temporal instability is present when the real part of $\sigma$ is positive for one or more values of $k\left(\sigma_{r}=\Re(\sigma)>0\right)$. We have validated our results via comparisons with [29] for several values of $R e$ with $\mathcal{D}=0$, and the overall approach used here is very similar to that used by Tseluiko et al.[29]. The main differences are that their study considered Newtonian fluids and allowed for spatiotemporal instability. 


\section{B. Numerical results}

Computed results indicate that the influence of wall topography can be stabilizing or destabilizing depending on the details of the deformation. Eigenspectra for $\mathcal{D}=0.32, S=$ $300, C_{t}=1$, and $L=15$ are shown in figure 3 a for $\delta=0,0.15$, and 0.3 . The flat-wall case is stable, and introducing a wall deformation with sufficiently large amplitude destabilizes the film flow via a Hopf bifurcation. Increasing the amplitude further introduces instability over a broader range of wavenumbers while also increasing the growth rates. The opposite trend is seen when $L=25$ and $\mathcal{D}=0.4$ (figure $3 \mathrm{~b}$ ). The flat-wall case is now linearly unstable and wall deformations tend to stabilize the flow. Similar trends are seen in calculations of
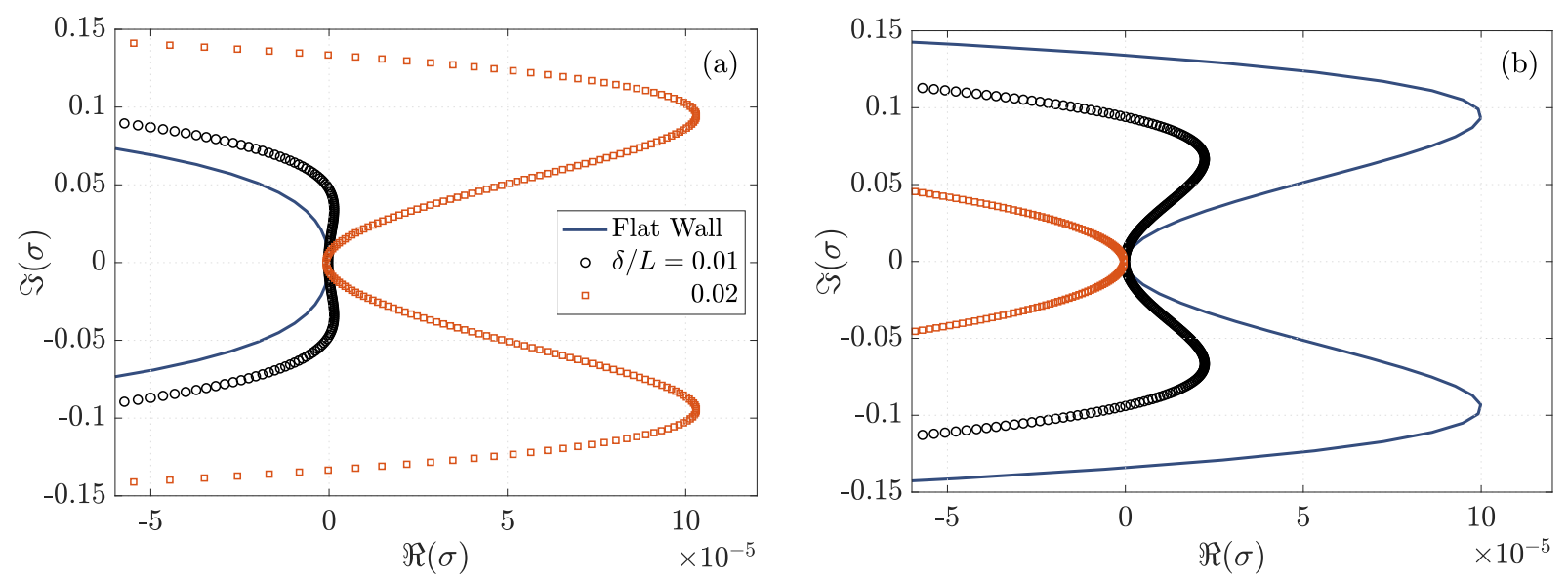

FIG. 3: Linear stability results, $S=300, C_{t}=1, R e=0$; eigensepectra for (a) $\mathcal{D}=0.32$,

$$
L=15 \text { and (b) } \mathcal{D}=0.4 \text { and } L=25 \text { (same legend as (a)). }
$$

the critical elasticity parameter, $\mathcal{D}_{\text {crit }}$ (figure $4 \mathrm{a}$ ). For the two smaller wavelengths shown, topography reduces $\mathcal{D}_{\text {crit }}$ and is destabilizing (instability occurs when $\mathcal{D}>\mathcal{D}_{\text {crit }}$ ) whereas the topography is stabilizing for $L=25$ and $L=40$. Again, increasing the deformation amplitude increases the influence of the topography, however, as can be seen in the figure, the sensitivity to the amplitude is very much dependent on the wall wavelength. A clearer view of these trends is found by simultaneously varying the wall wavelength and amplitude such that $\delta / L$ is held fixed. This constrains the slope of the wall topography, and the results for $\mathcal{D}_{\text {crit }}$ with $S=300$ and $C_{t}=1$ are shown in figure $4 \mathrm{~b}$ for $\delta / L=0.01,0.015$, and 0.02 . The figure shows there is an intermediate range of wavelengths, $10 \lesssim L \lesssim 40$, where the flow 

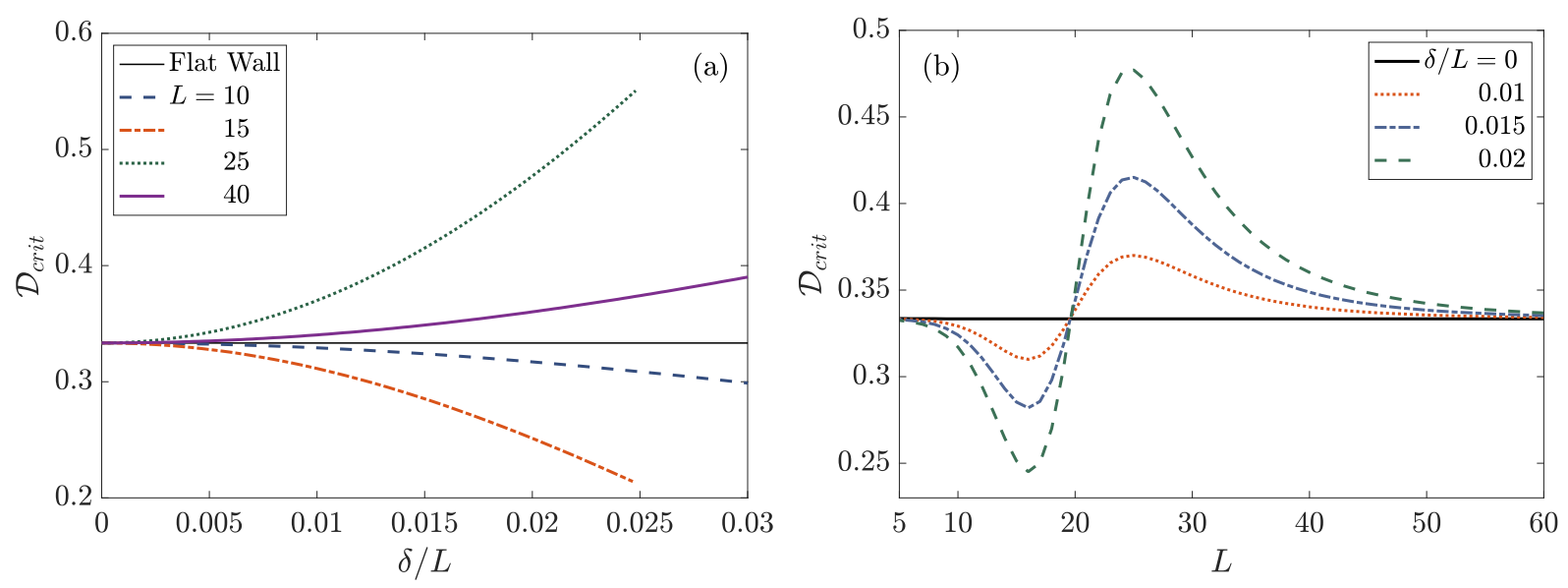

FIG. 4: Influence of topography on $\mathcal{D}_{\text {crit }}, S=300, C_{t}=1, R e=0$; individual curves correspond to (a) fixed wall wavelength, $L$, and (b) fixed wall steepness, $\delta / L$.

stability is particularly sensitive to topography. Within this intermediate range, topography initially exhibits a destabilizing influence reducing the critical Deborah number by more than $25 \%$ when $L=16$ and $\delta=0.32$. As the wavelength increases there is a rapid shift and topography becomes stabilizing, increasing $\mathcal{D}_{\text {crit }}$ by more than $40 \%$ for the parameters shown. For wavelengths outside of this range (both longer and shorter), the neutral curves are relatively insensitive to the wall deformation and, for these deformation amplitudes, $\mathcal{D}_{\text {crit }}$ remains close to its flat-wall value. The full eigenvalue spectra for three different wall wavelengths are shown in figure 5 a along with the spectrum for the flat wall case. Here, $\delta / L=0.02, \mathcal{D}=0.5$, and all cases shown are linearly unstable. The qualitative trends are similar to those seen for $\mathcal{D}_{\text {crit }}$ for $15 \leq L \leq 24$. There is a clear reduction in the growth rate as the wavelength increases, and the influence of topography shifts from destabilizing to stabilizing.

The most-unstable (or least-stable) modes correspond to particular values of the wavenumber, $k$, however, these wavenumbers do not provide a full picture of a mode's streamwise dependence which is of the form, $\exp \left(i k x_{1}\right) g\left(x_{1}\right)$. An effective wavenumber can be computed by applying a Fourier transform $\left(x_{1} \rightarrow \hat{k}\right)$ and then extracting the wavenumber at which the amplitude of the Fourier transform is maximum. These wavenumbers, $\hat{k}_{\max }$, are shown in figure 5 b with $\mathcal{D}=0.5$. For flat walls, $\hat{k}_{\max }=\sqrt{3 /(2 S)\left(6 / 5 R e+3 \mathcal{D}-C_{t}\right)}$, and topography substantially modifies the instability wavelength in the intermediate range of wall wave- 

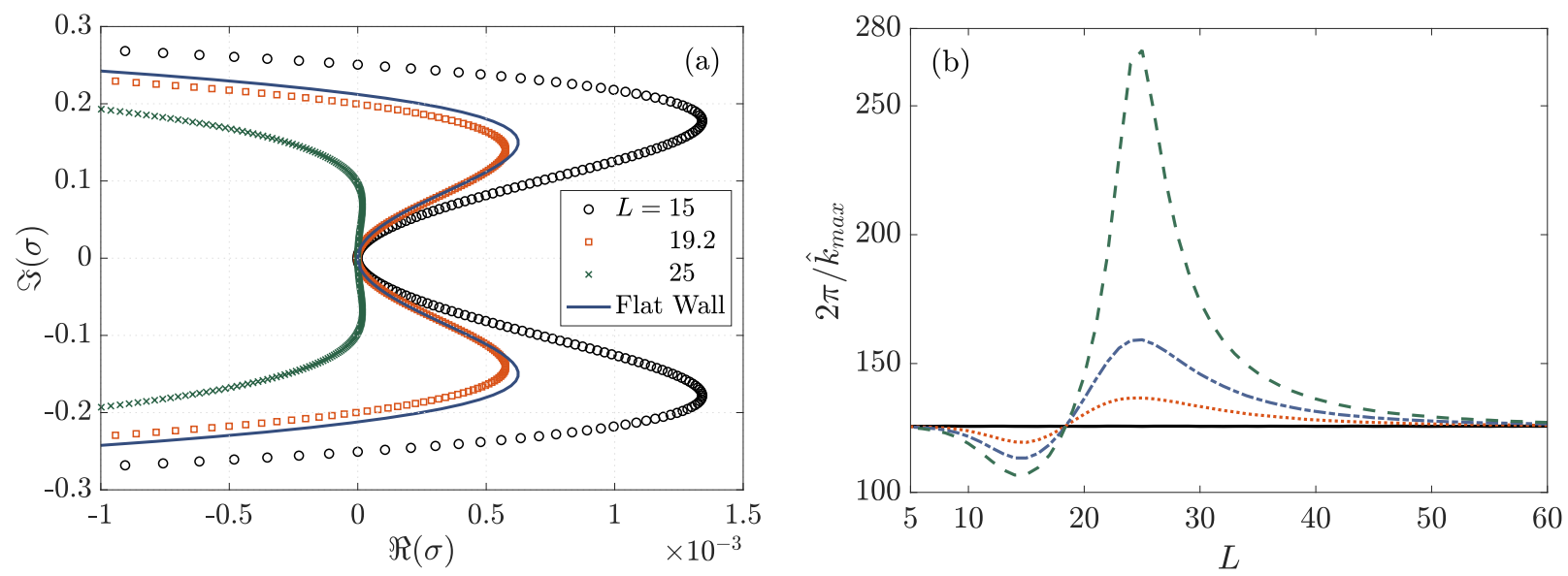

FIG. 5: Linear stability results, $S=300, C_{t}=1, R e=0$; (a) Eigenspectra for $\mathcal{D}=0.5$ and $\delta / L=0.02$; (b) Effective wavenumber for most destabilising mode (same legend as figure 4b)

lengths. Within the destabilizing range of wall wavelengths, the instability wavelengths are reduced, while in the stabilizing range, the wavelengths are substantially increased. Similar trends are observed for the effective wavenumbers of neutral waves computed with $\mathcal{D}=\mathcal{D}_{\text {crit }}$ (not shown).

\section{Influence of inclination angle and surface tension}

The results above all correspond to fixed values of the inclination $\left(C_{t}=1\right)$ and surface tension parameters $(S=300)$. Similar qualitative trends are observed when these parameters are varied. As discussed earlier, the critical elasticity parameter for a flat wall is, $\mathcal{D}_{\text {crit }}=C_{t} / 3$, so a flat vertical wall $\left(C_{t}=0\right)$ is linearly unstable with any non-zero Deborah number, and reducing the inclination angle from $90^{\circ}$ has a simple stabilizing effect. This stabilizing influence is also present for walls with topography as can be seen in figure 6a where $\mathcal{D}_{\text {crit }}$ curves shift upwards as $C_{t}$ is increased. Figure 6a additionally shows that there is typically a particular wavelength where the critical elasticity is approximately independent of $\delta$ and equal to its flat-wall value. It appears likely that the base-state phase difference plays an important role (see discussion below), however we have not been able to pinpoint the physical mechanism responsible for this behavior. These results also suggest that it is surface tension 
that dictates the range of wall wavelengths that influences $\mathcal{D}_{\text {crit }}$. Indeed, the results shown in figure $6 \mathrm{~b}$ confirm that the intermediate range of wavelengths is sensitive to $S$. Decreasing $S$ shifts this range to smaller wavelengths, and also reduces the magnitude of the influence of the wall topography. This shift is connected to trends in the base state shown in figure $2 \mathrm{~d}$. There, we see that decreasing the surface tension results in a more rapid "transition" from a flat free surface to one that is sinusoidal and synchronized with the wall. In figure $6 c, \mathcal{D}_{\text {crit }}$ is plotted against the base-state phase difference rather than L, and this removes the shift which indicates it is the influence of $S$ on the base-state phase difference which dictates the range of $L$ where $\mathcal{D}_{\text {crit }}$ is sensitive to the wall topography. The magnitude of this sensitivity is related linearly to $S$. This can be seen in $6 \mathrm{~d}$ where plotting the scaled critical elasticity, $\mathcal{D}_{\text {crit,scaled }}=\left(\mathcal{D}_{\text {crit }}-1 / 3\right) /(S / 100)$, against the phase difference nearly collapses the three curves. These results also show that with the addition of wall topography, surface tension no longer has a simple stabilizing effect and in fact can be destabilizing for certain wall wavelengths.

\section{Influence of topography on instability mechanism}

A number of studies have examined the linear instability mechanisms of gravity-driven films on flat walls (e.g. $[14,15,38,39])$. Huang \& Khomami[15] in particular provide a broad discussion of both inertial and elastic instability mechanisms in single and multilayer gravity-driven films. Their analysis of elastic instability in single-layer film flow utilized three different approaches. First, long-wave analysis was used to show that elastic stresses tend to 'push' fluid horizontally toward regions beneath crests in the perturbed free surface thus driving instability. This description is very similar to our discussion of the influence of elasticity in section II. Their second approach was based on analysis of a disturbance energy equation and again highlighted the importance of elastic stresses. Specifically it was found that elastic stresses acting at the free surface were primarily driving the instability. Finally, they examined the phase difference between the perturbed free surface and the disturbance vorticity, and verified that instability arises as this phase difference becomes positive. This corresponds to a positive induced vertical perturbation velocity at the crest of the disturbed free surface which is a destabilizing mechanism highlighted in our discussion below. 

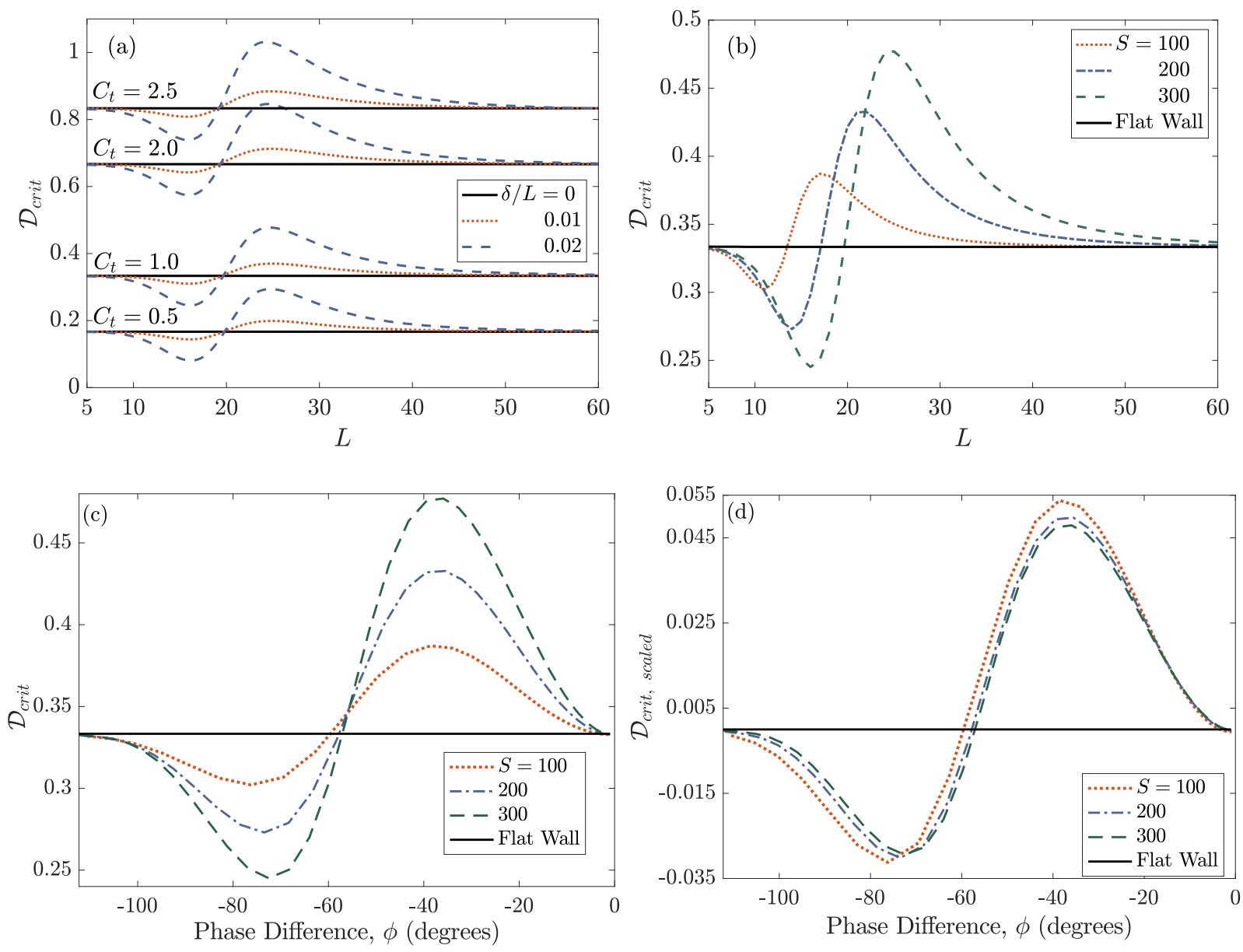

FIG. 6: Influence of $C_{t}$ and $S$ on $\mathcal{D}_{\text {crit }}, R e=0, \delta / L=0.02$; (a) $S=300$; (b) $C_{t}=1$; (c) $C_{t}=1, \mathcal{D}_{\text {crit }}$ vs. base-state phase difference, $\phi$; (d) $C_{t}=1, \mathcal{D}_{\text {crit,scaled }}$ vs. base-state phase difference, $\phi$.

The aim here is to elucidate how this elastic instability mechanism is modified by wall topography. Our analysis utilizes a disturbance 'energy' equation which is derived starting from the general linearized kinematic condition applied at the undisturbed free surface, $x_{2}=s+h_{0}$ (this follows directly from the linearization of (3)),

$$
\frac{\partial \eta}{\partial t}+\left[\left.\tilde{u}_{1}\right|_{f_{0}} \frac{\partial}{\partial x_{1}}\left(h_{0}+s\right)+\left.\bar{u}_{1}\right|_{f_{0}} \frac{\partial \eta}{\partial x_{1}}\right]=\left.\tilde{u}_{2}\right|_{f_{0}}+\left.\frac{\partial \bar{u}_{2}}{\partial x_{2}}\right|_{f_{0}} \eta
$$

where the velocity has been decomposed as $u_{i}=\bar{u}_{i}+\tilde{u}_{i}$, and ' $f_{0}$ ' indicates that a variable should be evaluated at $x_{2}=h_{0}+s$. General expressions for the velocities, $u_{i}^{(0)}\left(x_{2}, h\left(x_{1}\right) ; s\left(x_{1}\right)\right)$ and $u_{i}^{(1)}\left(x_{2}, h\left(x_{1}\right) ; s\left(x_{1}\right)\right)$, were obtained during the derivation of the VBE (see the appendix), and $u_{2}^{(2)}$ can be found from the continuity equation, $\partial u_{2}^{(2)} / \partial x_{2}=-\partial u_{1}^{(1)} / \partial x_{1}$. The base state 
velocities $\left(\bar{u}_{i}\right)$ are obtained by substituting $h=h_{0}$ into these expressions, and the perturbation velocities $\left(\tilde{u}_{i}\right)$ are then found by substituting equation (14) into the general expressions for the velocities, substracting the base state, and finally discarding terms which are, $O\left(|\eta|^{2}\right)$ or smaller. Defining $\hat{\eta}$ such that, $\eta=\hat{\eta}+\hat{\eta}^{*}$, equation 22 can then be rearranged into an 'energy-like' equation for $|\hat{\eta}|^{2}$,

$$
\begin{aligned}
\frac{\partial}{\partial t} \int_{0}^{L}|\hat{\eta}|^{2} d x_{1}= & -2 \int_{0}^{L}\left[\Re\left(\left.\hat{u}_{1}\right|_{f_{0}} \hat{\eta}^{*}\right) \frac{\partial}{\partial x_{1}}\left(h_{0}+s\right)\right] d x_{1} \\
& +2 \int_{0}^{L}\left[\Re\left(\left.\hat{u}_{2}\right|_{f_{0}} \hat{\eta}^{*}\right)+\left.\frac{\partial \bar{u}_{2}}{\partial x_{2}}\right|_{f_{0}}|\hat{\eta}|^{2}\right] d x_{1}-\left.\int_{0}^{L} \bar{u}_{1}\right|_{f_{0}} \frac{\partial|\hat{\eta}|^{2}}{\partial x_{1}} d x_{1}
\end{aligned}
$$

with $\hat{u}_{i}$ defined such that $\tilde{u}_{i}=\hat{u}_{i}+\hat{u}_{i}{ }^{*}$. From (19), we have $\hat{\eta}=\exp \left(\sigma t+i k x_{1}\right) g\left(x_{1}\right)$, and then letting $\left.\hat{u}_{i}\right|_{f_{0}}=\exp \left(\sigma t+i k x_{1}\right) v_{i}\left(x_{1}\right)$, equation (23) can be restated as:

$$
\begin{array}{r}
\sigma_{r}=\mathcal{P}_{1}+\mathcal{P}_{2}+\mathcal{P}_{3}, \\
\mathcal{P}_{1}=-\frac{1}{\mathcal{G}} \int_{0}^{L}\left[\Re\left(v_{1} g^{*}\right) \frac{\partial}{\partial x_{1}}\left(h_{0}+s\right)\right] d x_{1}, \\
\mathcal{P}_{2}=\frac{1}{\mathcal{G}} \int_{0}^{L}\left(\Re\left(v_{2} g^{*}\right)+\left.\frac{\partial \bar{u}_{2}}{\partial x_{2}}\right|_{f_{0}}|g|^{2}\right) d x_{1}, \\
\mathcal{P}_{3}=-\left.\frac{1}{\mathcal{G}} \int_{0}^{L} \frac{1}{2} \bar{u}_{1}\right|_{f_{0}} \frac{\partial|g|^{2}}{\partial x_{1}} d x_{1}=-\left.\frac{1}{\mathcal{G}} \int_{0}^{L} \frac{1}{2} \frac{\partial \bar{u}_{2}}{\partial x_{2}}\right|_{f_{0}}|g|^{2} d x_{1},
\end{array}
$$

where $\mathcal{G}=\int_{0}^{L}|g|^{2} d x_{1}$. For a flat wall, $\mathcal{P}_{1}$ and $\mathcal{P}_{3}$ are zero, and (24a) reduces to,

$$
\sigma_{r}=\frac{1}{\mathcal{G}} \int_{0}^{L} \Re\left(v_{2} g^{*}\right) d x_{1} .
$$

The growth rate then tends to be large when vertical velocity perturbations and the free-surface perturbation are in-phase with each other. This is a well-established picture of interfacial instability and is consistent with the Huang \& Khomami[15] description of elastic (and inertial) instability driven by vorticity perturbations inducing upward motion at the crests (and downward motion at troughs) of the perturbed free surface. With sinusoidal walls, the base-state free surface, $f_{0}\left(x_{1}\right)$, is no longer flat, and it is helpful to consider the perturbation velocity and displacement in the direction normal to $f_{0}$ (see figure 7 ). Let $\psi$ be the angle between this normal and the $x_{2}$ direction as indicated in the figure. The normal component of the perturbation velocity at the free surface is $\tilde{u}_{n}=\left.\tilde{u}_{1}\right|_{f_{0}+\eta} \sin \psi+\left.\tilde{u}_{2}\right|_{f_{0}+\eta} \cos \psi$. Since $\tan \psi=-d f_{0} / d x_{1}$, this can be rearranged as, $\tilde{u}_{n}=\cos \psi\left(-\left.\tilde{u}_{1}\right|_{f_{0}+\eta} d f_{0} / d x_{1}+\left.\tilde{u}_{2}\right|_{f_{0}+\eta}\right)$ 


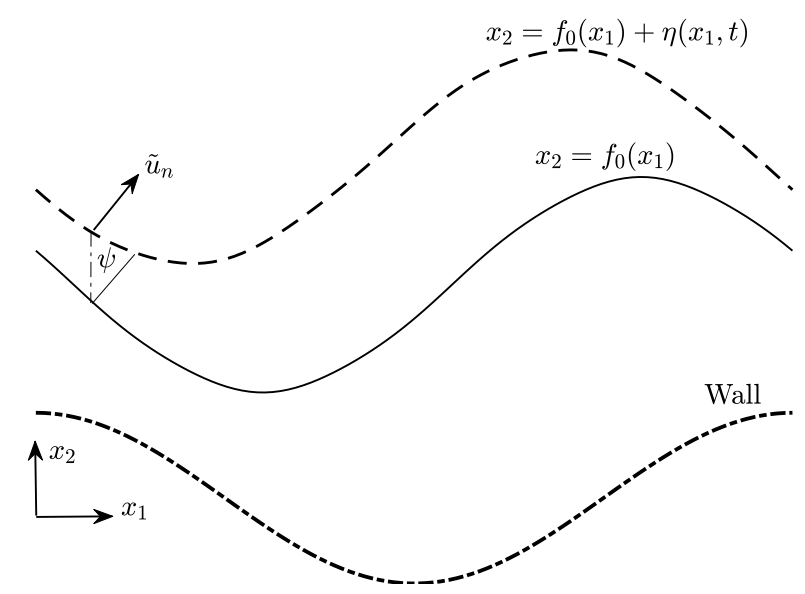

FIG. 7: Illustration of (local) destabilization driven by $\tilde{u}_{n}$ at $x_{2}=f_{0}+\eta\left(\tilde{u}_{n}\right.$ is the component of the perturbation velocity normal to the base-state free surface).

(cf. equation 22). Then, defining $v_{n}$ such that $\left.\tilde{u}_{n}\right|_{f_{0}+\eta}=v_{n} \exp \left(\sigma t+i k x_{1}\right)+c . c$. , it follows that,

$$
\mathcal{P}_{1}+\mathcal{P}_{2}=\frac{1}{\mathcal{G}} \int_{0}^{L} \Re\left(v_{n} g_{n}^{*}\right)\left[1+\left(\frac{d f_{0}}{d x_{1}}\right)^{2}\right] d x_{1},
$$

where $g_{n}=g \cos \psi$ is the projection of $g$ in the normal direction, and we have used, $1 / \cos ^{2} \psi=$ $1+\left(d f_{0} / d x_{1}\right)^{2}$. Noting that $\left(d f_{0} / d x_{1}\right)^{2} \sim O\left(\epsilon^{2}\right)$, we see that for thin films instability is enhanced when $g_{n}$ and $v_{n}$ are in phase with each other, and the combined effect of $\mathcal{P}_{1}$ and $\mathcal{P}_{2}$ can be interpreted as a generalization of (25) for thin-film flows with wall topography. The third term, $\mathcal{P}_{3}$, is related to the streamwise advection of the free-surface perturbation by the base state velocity, $\left.\bar{u}_{1}\right|_{f_{0}}$. For flat walls, $\left.\bar{u}_{1}\right|_{f_{0}}$ is constant, and the resulting simple translation of the perturbation has no effect on the growth rate $\left(\mathcal{P}_{3}=0\right)$. With the introduction of topography, the streamwise variation of $\left.\bar{u}_{1}\right|_{f_{0}}$ leads to local stretching and compression of $\eta$ in the streamwise direction. The global effect of these deformations on the growth rate can be interpreted using the second integral in (24d) (obtained using integration by parts). Comparing this integral with $\mathcal{P}_{2}$, we see that when $\mathcal{P}_{3}<0$, it directly counteracts any destabilization driven by $\tilde{u}_{2}$ at the perturbed free surface.

The influence of the three $\mathcal{P}$ terms is illustrated in figure 8a which shows the decomposition of $\sigma_{r}$ for the most-unstable mode with $\mathcal{D}=0.5, \delta / L=0.02$, and varying $L$. The growth rate follows the general trend seen for $\mathcal{D}_{\text {crit }}$ in figures $4 \mathrm{~b}$ and 6 above. Initially, at smaller wall wavelengths, the instability is unaffected by topography. Surface tension tends to 'flatten' 
the steady-state free surface, and as a result, $\mathcal{P}_{1}, \partial \bar{u}_{2} / \partial x_{2}$, and $\mathcal{P}_{3}$ all tend to be small, and the perturbation dynamics near the free surface remain essentially the same as in the case of a flat wall. For large wavelengths $(L \gtrsim 40)$, the instability mechanism again matches results for a flat wall. At these wavelengths, the steady-state film thickness tends towards a constant value, so the integrand in (24b) becomes $\pi \delta / L \Re\left(v_{1} g^{*}\right) \sin \left(\kappa x_{1}\right)$. Integration over a wall period will only be non-zero if $\Re\left(v_{1} g^{*}\right)$ contains non-zero Fourier components with wavenumbers, $\pm \kappa$. In practice for the parameters considered here, these components for the most unstable waves tend to be small, and thus for larger values of $L, \mathcal{P}_{1}$ tends to be small. The third term, $\mathcal{P}_{3}$ also goes to zero at larger wavelengths, and this behavior can be understood by examining $\partial \bar{u}_{1} / \partial x_{1}$ since $\mathcal{P}_{3}$ will be zero when $\bar{u}_{1}$ is constant as in the flat-wall case. Taking $h_{0}=1$, we find that $\partial \bar{u}_{1} / \partial x_{1}=\delta \pi^{2} / L \cos \left(\kappa x_{1}\right)\left(3 / L C_{t}+4 \pi^{2} / L^{3} S\right)$, and the discussion of the integrand for $\mathcal{P}_{1}$ can be applied here as well. Also note that if $\delta / L$ is held fixed and $L$ is increased (as in figure 8), then the amplitude of $\partial \bar{u}_{1} / \partial x_{1}$ will tend to decrease. The most interesting behavior is again in the intermediate range, $10 \lesssim L \lesssim 40$. Within this range, topography substantially modifies the perturbation field near the free surface. There is "improved" alignment between $g_{n}$ and $\tilde{u}_{n}$ as indicated by the larger values of $\mathcal{P}_{1}+\mathcal{P}_{2}$ relative to the growth rate for the flat wall case. However, this is balanced by the stabilizing influence of $\mathcal{P}_{3}$. Ultimately, there is a complex balance which leads to a destabilzing influence for $10 \lesssim L \lesssim 19$ and a stabilizing effect for $19 \lesssim L \lesssim 40$. Generally, instability is still driven by perturbation elastic stresses, however these results indicate that the coupling between these stresses, the free surface, and velocity changes tangibly. These qualitative trends remain the same when the inclination angle and elasticity are varied (figure $8 b)$. Furthermore, these trends are qualitatively similar but smaller in magnitude at smaller $S$ (not shown). Figure 9 focuses on the most unstable mode at $\mathcal{D}_{\text {crit }}+0.005$ and tracks its growth rate and the $\mathcal{P}$ terms as the Deborah number is varied. This provides further insight into the influence of topography near the onset of instability (it is difficult to track the neutral mode which is why the most unstable mode at $\mathcal{D}_{\text {crit }}+0.005$ has been chosen). For both wavelengths, the growth rate increases with $\mathcal{D}$, however the relatively small values of $\mathcal{P}_{1}$ and $\mathcal{P}_{2}$ delay the onset of instability for $L=25$. The rate of increase of the growth rate is higher for $L=25$ and with sufficiently large $\mathcal{D}$, this case has a higher growth rate than both the flat wall and $L=15$ cases. It follows that the descriptions above of wall-wavelengths as 

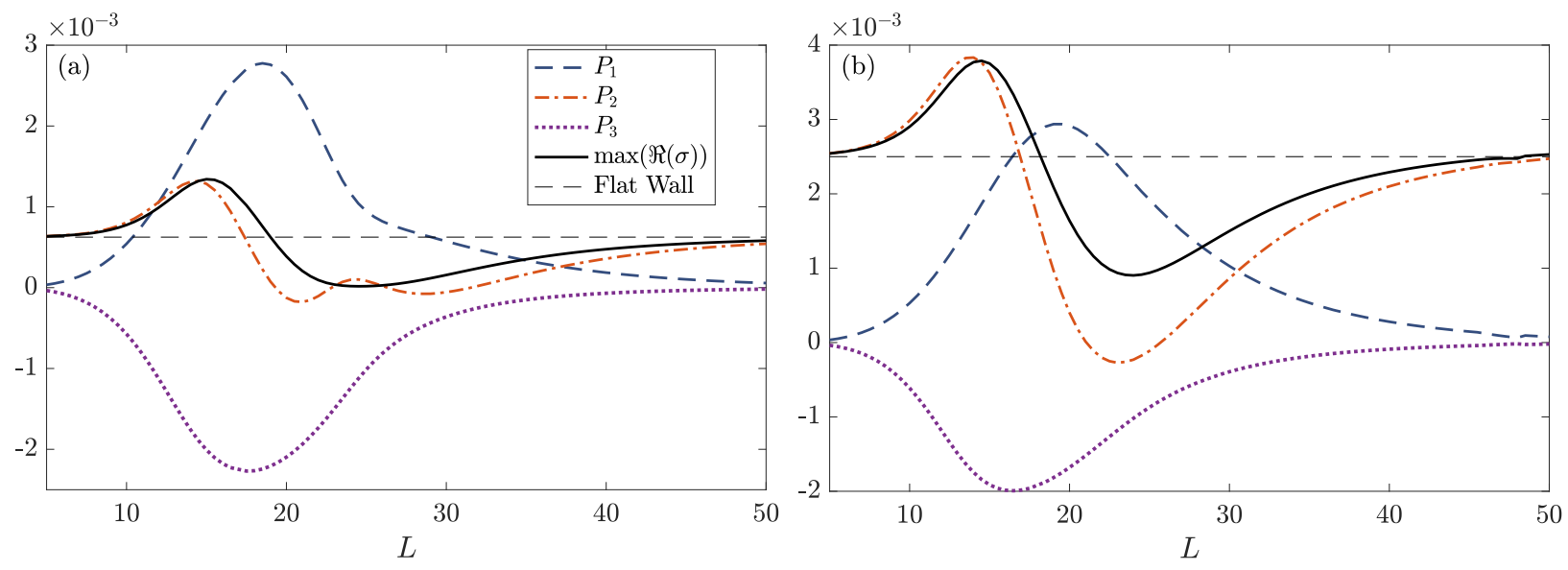

FIG. 8: Decomposition of maximum growth rate, $\max (\Re(\sigma))$ of elastic instability, $R=0$, for various wall wavelengths with $S=300, \delta / L=0.02$ : (a) $\mathcal{D}=0.5, C_{t}=1.0$; (b) $\mathcal{D}=1$, $C_{t}=2.0$ (same legend as figure (a)).
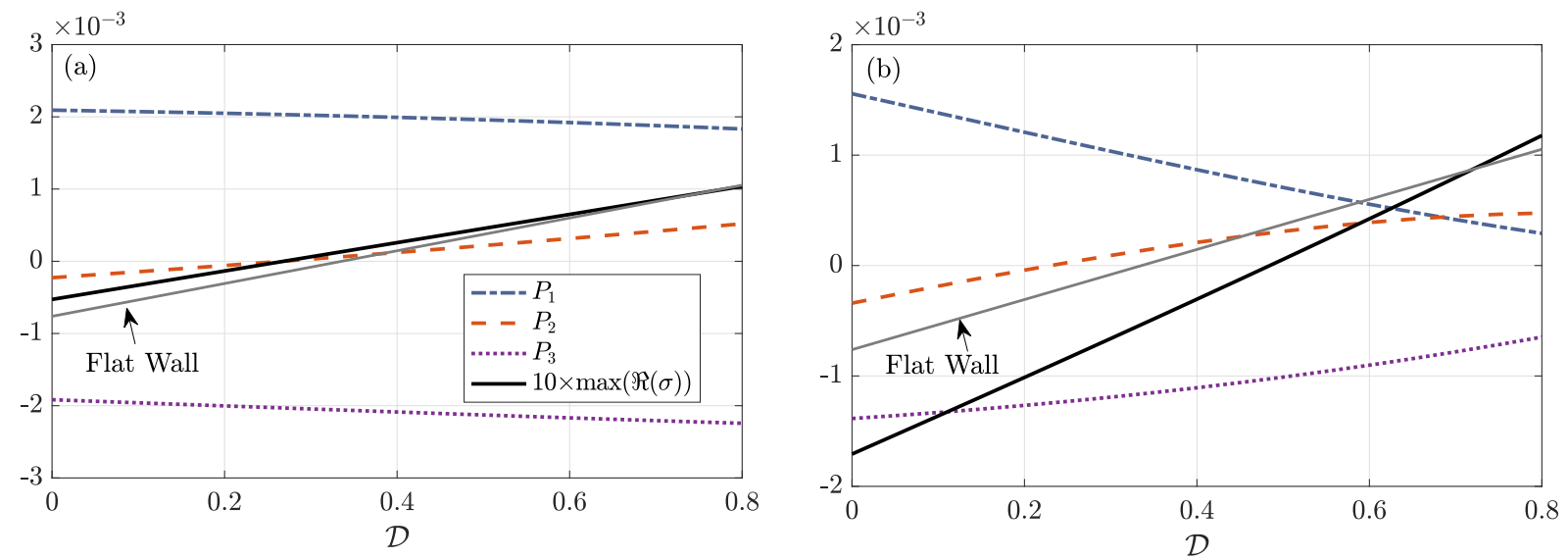

FIG. 9: Decomposition of growth rate, corresponding to most unstable mode at $0.005+$ $\mathcal{D}_{\text {crit }}, \max \left(\Re(\sigma)_{\text {crit }}\right)$, with, $C_{t}=1, S=300, \delta / L=0.02$ : (a) Destabilising wall, $L=15$ $\left(\mathcal{D}_{\text {crit }}=0.25\right)$; (b) Stabilising wall, $L=25\left(\mathcal{D}_{\text {crit }}=0.47\right.$, same legend as (a)).

stabilizing or destabilizing should only be applied near the onset of linear instability. 


\section{Inertial instability}

The qualitative trends shown in figure 4a have also been observed in studies of Newtonian film flows on sinusoidal walls[26, 27, 29], which showed that topography could increase or reduce the critical Reynolds number. Here, we present linear stability results which allow direct comparisons between Newtonian and inertialess (elastic) flows. With a flat wall, inertia and elasticity enter the linearized equations (17) as the sum, 6/5Re $+3 \mathcal{D}$, and an inertialess flow with $\mathcal{D}=\mathcal{D}_{0}$ exhibits identical linear dynamics to a Newtonian flow with $R e=5 / 2 \mathcal{D}_{0}$. This formal equivalence between the effects of inertia and elasticity is no longer present when topography is introduced, however the numerical results in figure 10 show strong qualitative agreement between $\mathcal{D}_{\text {crit }}$ and $2 / 5 R e_{\text {crit }}$ for a range of values of $S$ and $C_{t}$. In fact, there is clear quantitative agreement over a broad range of parameters with differences appearing at larger wavelengths and smaller inclinations. The influence of topography is stronger (destabilising) for the Newtonian cases in this parameter range, and the $R e_{\text {crit }}$ curves only return to their flat-wall values at very large wavelengths which are not shown in the figure. The decomposition of the growth rate, equation (24), shown in figure 11 follows the same qualitative trends discussed for elastic instabilities above. Streamwise variations of the base state at the free surface induced by topography again play a key role in modifications to the most-unstable and neutral modes within an intermediate range of wall wavelengths.

\section{NON-LINEAR DYNAMICS}

A number of studies have investigated the evolution of nonlinear waves on thin films overlying both flat and sinusoidal walls. Oron \& Gottlieb[20] provide a systematic numerical study of the Benney equation subject to periodic boundary conditions and reported stationary and time-periodic traveling waves as well as non-stationary waves (which constantly change their shape with time). Trifonov[40] performed a numerical investigation of the Navier-Stokes equations for Newtonian fluids to specifically obtain travelling wave solutions and compared flow over sinusoidal walls to that over a flat wall. At the lowest Reynolds number considered in that study, $R e=20 / 3\left(C_{t}=\cot \left(10^{\circ}\right) \approx 5.67, S=6.62\right)$, the free surface disturbance for a flat wall consisted of asymmetric, non-linear humps characterised by a 

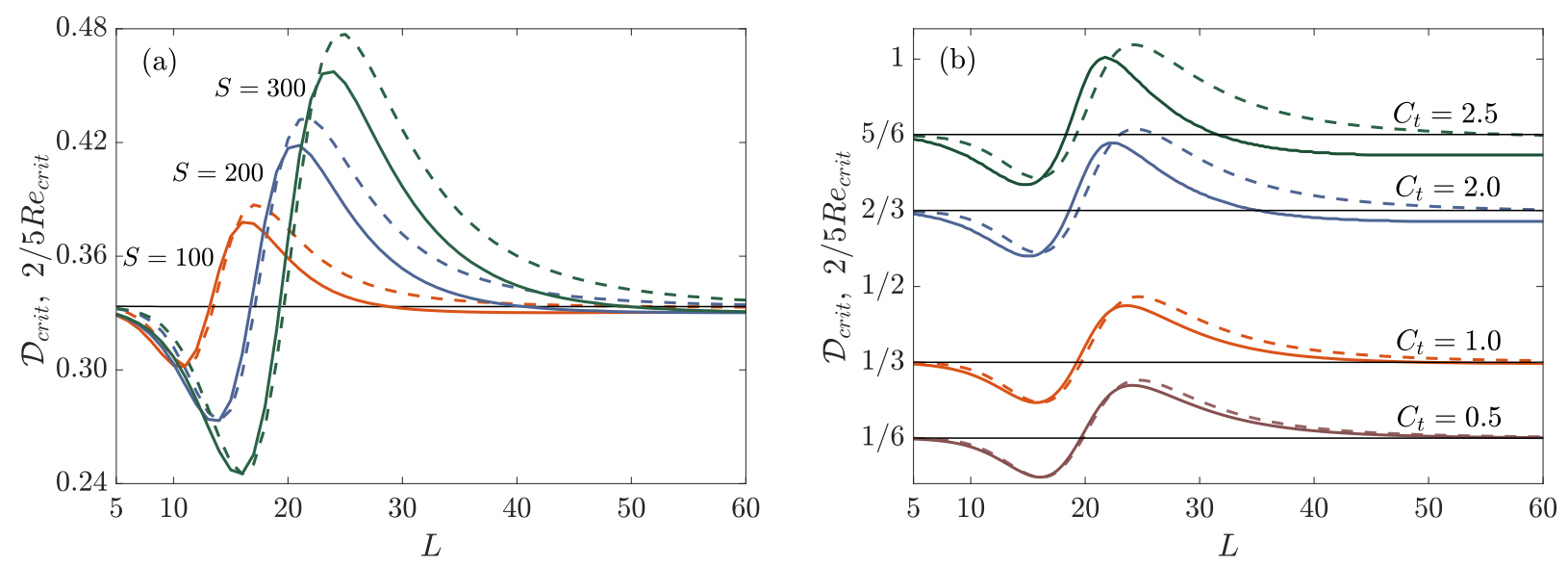

FIG. 10: Linear stability results, $\delta / L=0.02$ :

Critical inertial (for $\mathcal{D}=0$ ) and - elastic (for $R e=0$ ) instability for various values of (a) $S$ with $C_{t}=1$; (b) $C_{t}$ with $S=300$.
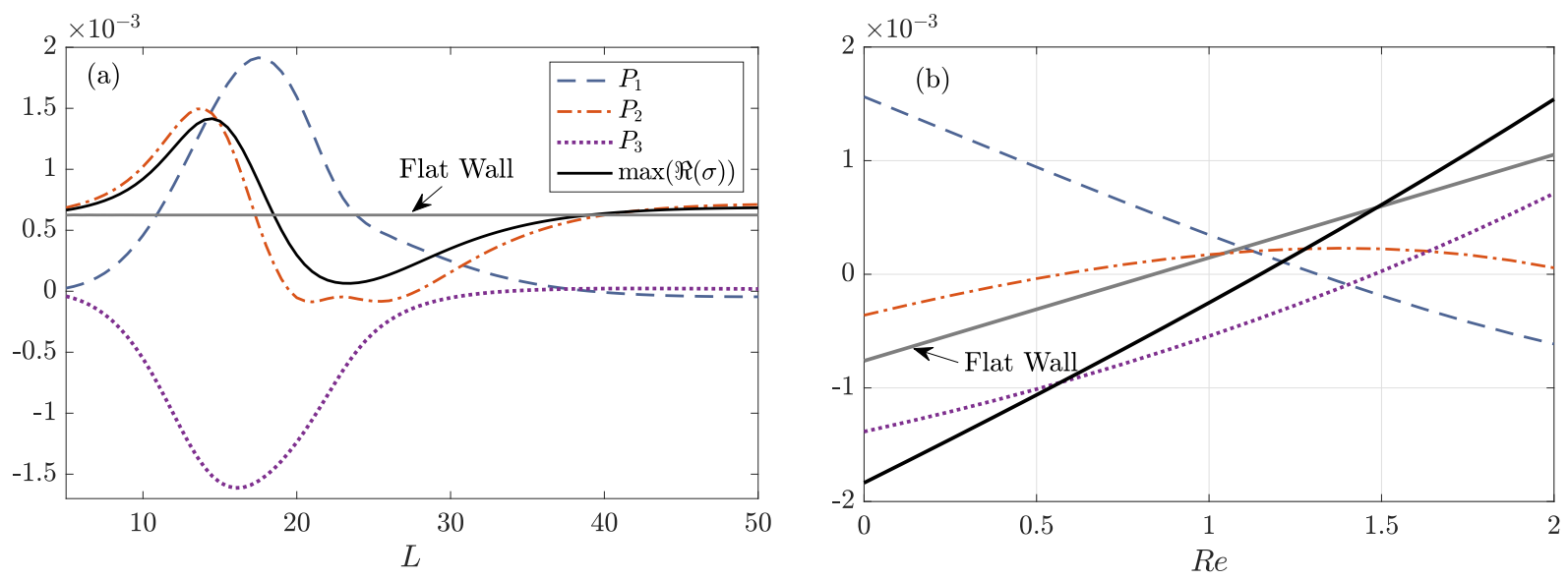

FIG. 11: Decomposition of growth rate for Newtonian films with $\mathcal{D}=0, C_{t}=1, S=300$, $\delta / L=0.02$ : (a) most-unstable mode, $R e=1.25$, varying $L$; (b) near-neutral mode, $L=25$, varying $R e$.

relatively sharp front and a long, smooth tail. Introduction of a sinusoidal wall $(\delta / L=0.4)$ modified these disturbances to include finer spatial scales: shorter waves overlaid the humps so these modified travelling waves were doubly periodic in space. Similar waves had been observed earlier in the experiments of Reck \& Aksel[41]. Oron \& Heining[25] derived a weightedresidual IBL model and also simulated unsteady films on sinusoidal walls. They indentified a range of nonlinear states similar to those observed for flat walls. Dávalos-Orozoco[28] 
investigated the Benney equation forced with a time-periodic, spatially-localized pressure perturbation. The perturbations excited by this forcing were suppressed when the steady state free surface consisted of deep valleys; therefore suitable wall wavelengths could be chosen that stabilised the flow. This approach was applied to Oldroyd-B fluids in [1] which showed that elastic instability could be similarly suppressed. However, the non-linear dynamics of the disturbance evolution have not been fully characterized for the VBE.

Here, we present results from extensive numerical simulations of the VBE (equation (5)) and provide a broad view of the influence of sinusoidal topography on the nonlinear states generated by elastic instability. We will compare results for flat walls with sinusoidal walls with $L=15,25$, and 150 which correspond to destabilizing, stabilizing, and neutral (growth rate is unaffected) wavelengths. The results below illustrate the influence of elasticity and topography on the bifurcation structure and amplitudes of nonlinear waves on viscoelastic films.

\section{A. Numerical Method}

We consider the evolution of the film thickness, $h\left(x_{1}, t\right)$ and, in particular, the perturbation, $\zeta\left(x_{1}, t\right)$ where, $h=h_{0}\left(x_{1}\right)+\zeta$, and $h_{0}$ is the pre-computed steady state. The initial perturbation is,

$$
\zeta\left(x_{1}, 0\right)=10^{-3} \cos \left(\frac{2 \pi x_{1}}{L}\right) \exp \left(-\frac{\ln 2}{L_{\text {gauss }}^{2}} x_{1}^{2}\right), \quad L_{\text {gauss }}=\frac{L_{D}}{15},
$$

where $L$ is again the wall wavelength, $L_{D}=N_{\text {periods }} L$ is the length of the computational domain, and we will set $L_{D}=150$ and $\delta=0.02$ (for flat walls, we set $L=L_{D}$ ). We use a pseudospectral approach where spatial derivatives are calculated using fast Fourier transforms, and the time integration is performed using Matlab's multi-step, variable-order solver, ode15s[35]. The code has been validated via comparisons with linear stability computations and by reproducing results for Newtonian films on flat walls in Oron \& Gottlieb[20]. The analysis of our numerical results focuses on the perturbation 'energy',

$$
E(t)=\frac{1}{L_{D}} \int_{0}^{L_{D}} \zeta\left(x_{1}, t\right)^{2} d x_{1}
$$

and we use maps of its local extrema, $E^{*}$, to (partially) characterize the nonlinear dynamics. A similar approach was used in [42]. 

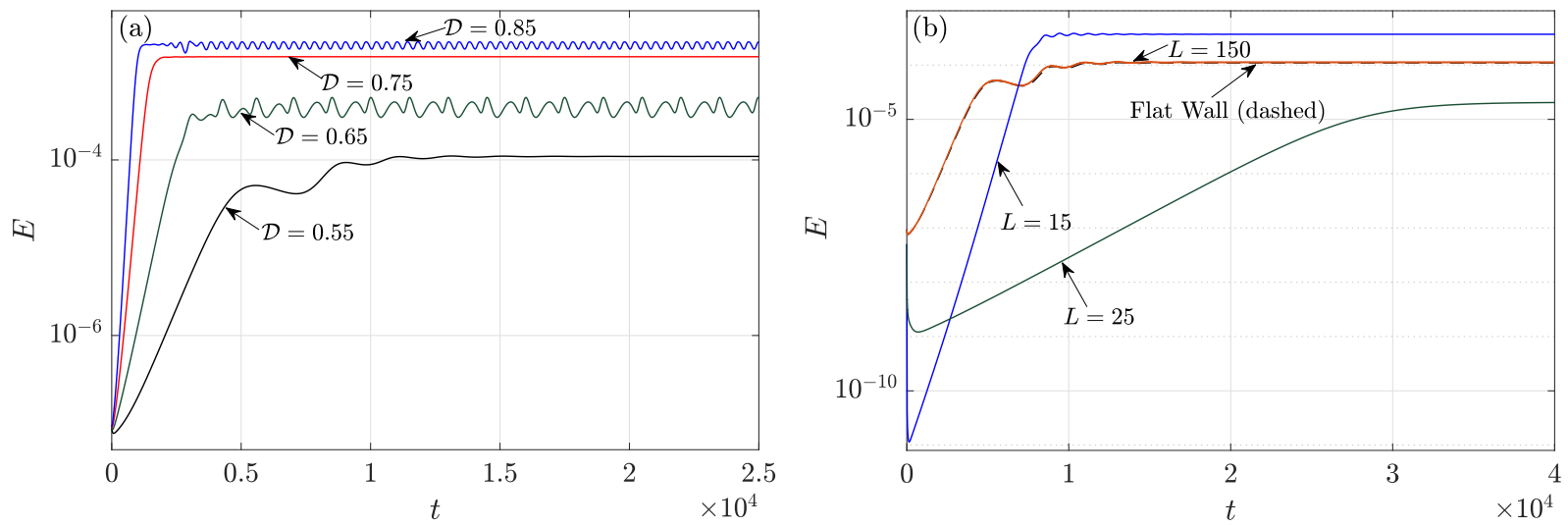

FIG. 12: Evolution of disturbance energy for $C_{t}=1, S=300, R e=0, L_{D}=150$ and $\delta / L=0.02$ : (a) Flat wall, varying $\mathcal{D}$; (b) $\mathcal{D}=0.55$, different walls.

\section{B. Inertialess films}

We begin with a flat wall and set $S=300, C_{t}=1$, and $R e=0$. Linear instability first occurs for this finite-sized computational domain at $\mathcal{D}_{\text {crit }}=0.39$ (with wavenumber $k=2 \pi / 150$ ), and the evolution of the energy at four supercritical values of $\mathcal{D}$ is shown in figure 12a. Initially, there is a very short transient 'adjustment' where the energy decreases as linearly stable components of the initial perturbation rapidly decay. Following this adjustment, there is clear exponential growth. Eventually, the perturbation saturates due to nonlinearity, and we see three nonlinear states which can be characterized by their temporal dynamics: 1$)$ travelling waves $(\mathcal{D}=0.55$ and 0.75 , characterized by a constant energy level), 2) period $-1(\mathcal{D}=0.85)$, and 3$)$ period $-2(\mathcal{D}=0.65)$ solutions. The latter two states arise via period-doubling subharmonic bifurcations (see section VB). Also, the mean energy level of the final nonlinear state increases with elasticity. The spatio-temporal evolution of the non-linear states beyond the initial transient time, $t_{\text {transient }}=2 \times 10^{5}$ for $\mathcal{D}=0.55$ and $\mathcal{D}=0.85$ is shown in figures $13 \mathrm{a}$ and $13 \mathrm{e}$ respectively. Here the major streamwise translational component of the perturbation is removed by transforming the streamwise direction, $x$, to a moving frame, $x-c \cdot \tau$, where $c \approx 3$ and $\tau=t-\left(t_{\text {transient }}+t_{0}\right)$. The parameter $t_{0}$ is set to align the solutions for illustrative purposes. The travelling wave solution in figure 13a has a similar characteristic shape to that observed in [40]. The time periodic (period-2) evolution of the perturbation for $\mathcal{D}=0.85$ can also be observed in figure $13 \mathrm{e}$. 

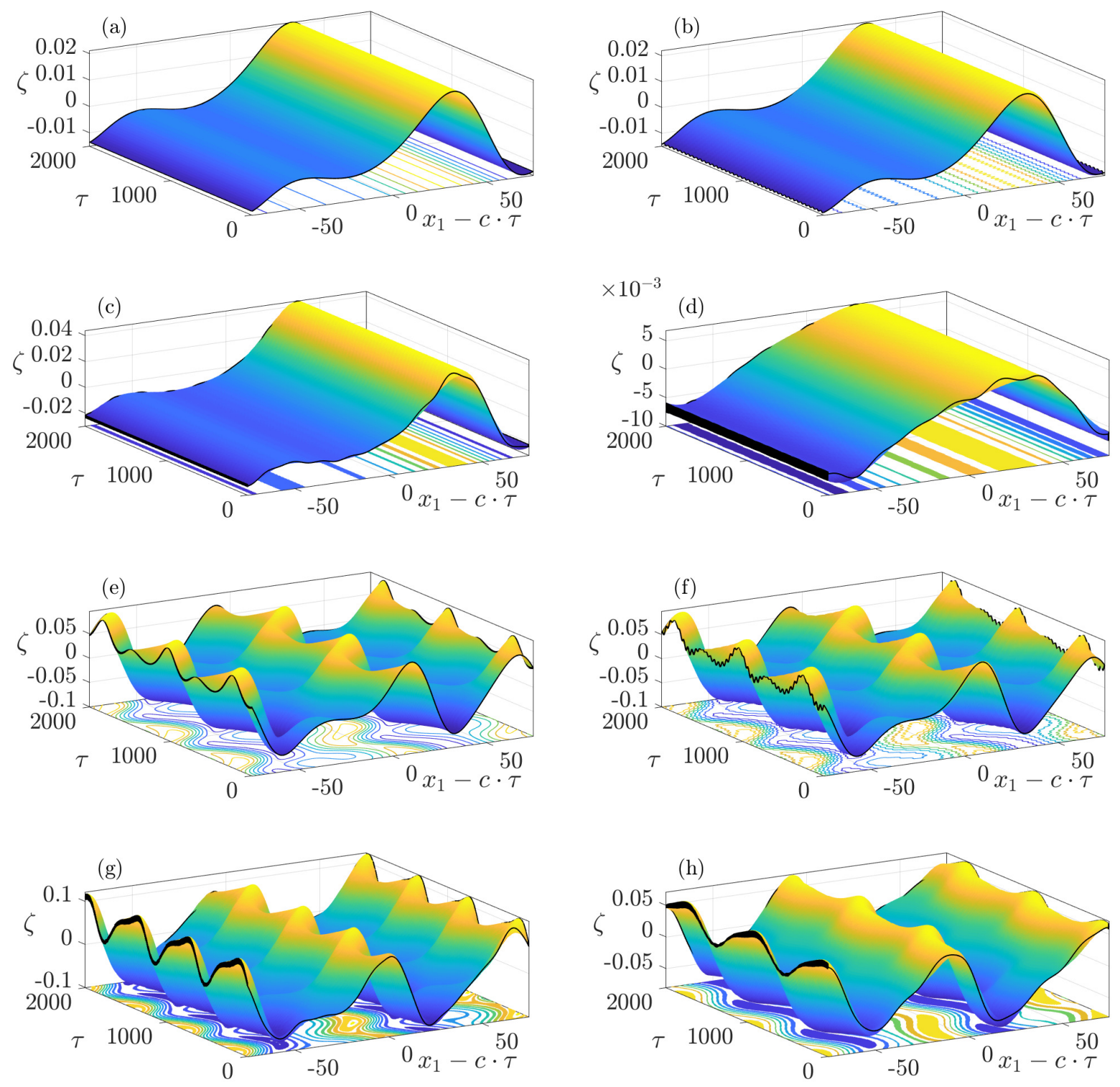

FIG. 13: Spatio-temporal evolution of the perturbations $\zeta$, for $C_{t}=1, S=300, R e=0$, $L_{D}=150$ and $\delta / L=0.02\left(\tau=t-\left(t_{0}+2 \times 10^{5}\right)\right): \mathcal{D}=0.55-\left(\right.$ a) Flat $\left(t_{0}=25, c=3.022\right)$, (b) $L=150\left(t_{0}=20, c=3.021\right)$, (c) $L=15\left(t_{0}=33.5, c=2.964\right)$ and (d) $L=25\left(t_{0}=45\right.$, $c=3.081)$ sinusoidal walls; $\mathcal{D}=0.85-(\mathrm{e})$ Flat $\left(t_{0}=0, c=3.025\right)$, (f) $L=150\left(t_{0}=31\right.$, $c=3.023),(\mathrm{g}) L=15\left(t_{0}=137.5, c=2.969\right)$ and $(\mathrm{h}) L=25\left(t_{0}=35, c=3.051\right)$ sinusoidal walls.

Now, we move on to the effect of sinusoidal topography. Replacing the flat wall with a $L=150$ sinusoidal wall has no tangible effect on the linear dynamics (section IV and figure 

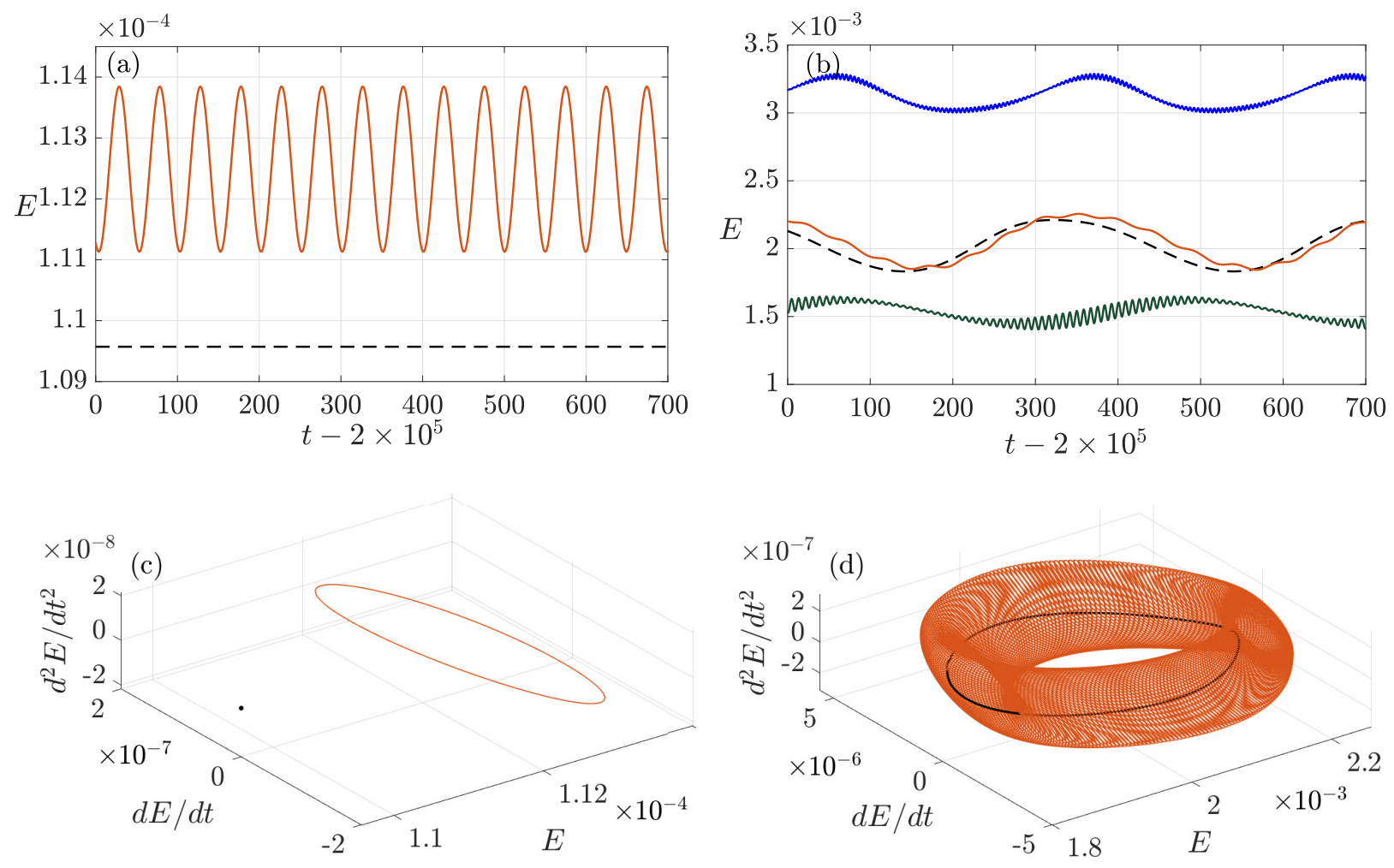

FIG. 14: Temporal evolution of $E$ of the saturated non-linear state for $C_{t}=1, R e=0$, $S=300, L_{D}=150$ and $\delta / L=0.02$ with (a) $\mathcal{D}=0.55$ and (b) $\mathcal{D}=0.85$ and their representation in 3D energy phase space in (c) and (d) respectively (for $\left.0 \leq t-2 \times 10^{5} \leq 5 \times 10^{4}\right)$ for flat (- - ) and $L=150$ ( $(-)$ sinusoidal walls. In figure (b) $E$ for $L=15$ (destabilising wall, $\longrightarrow$, top curve) and $L=25$ (stabilising wall, $\square$, bottom curve) sinusoidal walls respectively are also shown.

12b), however the nonlinear state exhibits finer temporal scales for both $\mathcal{D}=0.55$ and 0.85 . The latter can be observed both in the energy plots (at large times) shown in figure 14a and 14b and the space-time plots in figure 13 (compare figure 13b with 13a and 13f with 13e). This effect can also be described geometrically: the attractor for the flat wall dynamics lie is modified into a higher dimensional object for the $L=150$ sinusoidal wall as shown in the 3D $\left(E-\mathrm{d} E / \mathrm{d} t-\mathrm{d}^{2} E / \mathrm{d} t^{2}\right)$ phase portraits in figures $14 \mathrm{c}$ and $14 \mathrm{~d}$. A travelling wave (represented by a point attractor in the energy phase space) for $\mathcal{D}=0.55$ is modified into a period -1 solution (a single loop in the 3D phase space), and the period-1 solution for $\mathcal{D}=0.85$ becomes modulated by smaller waves resulting in the formation of dense tranjectories on 

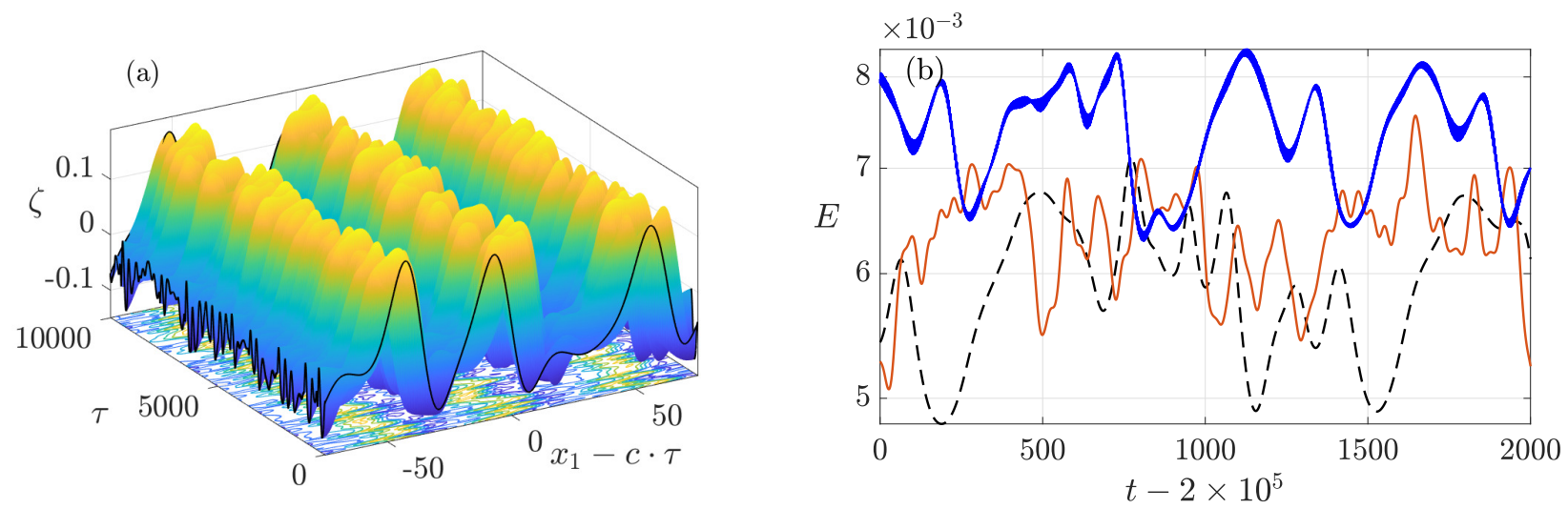

FIG. 15: Dynamics of the saturated non-linear state for $C_{t}=1, R e=0, S=100$, $L_{D}=150, \mathcal{D}=0.85$ and $\delta / L=0.02$ : (a) Spatio- temporal evolution of the perturbation $\zeta$ $\left(x, \tau=t-2 \times 10^{5}\right)$ for flat wall; (b) temporal evolution of $E$ (same legend as figure 14b but without the stabilising wall; the destabilising wall corresponds to $L=10$ ).

the surface of a torus in the 3D phase space and suggesting quasi-periodic dynamics. These changes are distinct from those reported by Trifonov[40] where travelling wave solutions acquired additional spatial periods with the introduction of the sinusoidal wall.

Results for $L=15$ and 25 are also included in figure $12 \mathrm{~b}$. We see that the stabilizing wall $(L=25)$ has a smaller growth rate and saturates at a lower mean energy relative to the flat wall while the destabilizing wall $(L=15)$ results correspond to a larger growth rate and a higher mean energy. These smaller- $L$ cases also produce fine temporal scales similar to those observed for $L=150$. The nonlinear states are quasi-periodic at $\mathcal{D}=0.55$ and 0.85 for both these walls, and the amplitude of fluctuations (figure 13) as well as the mean $E$ (12b and 14b) of the non-linear state of the disturbance decrease as we move from $L=15$ to $L=25$.

Reducing the surface tension to $S=100$ results in more irregular temporal dynamics and increases the amplitude of $\zeta$ as shown in the spatio-temporal evolution of the non-linear state for the flat wall with $\mathcal{D}=0.85$ in figure $15 \mathrm{a}$. Here, the temporal evolution is chaotic for the flat wall as also shown in the $E-t$ plot in figure 15b. Again, the introduction of topography produces finer temporal scales (figure 15b). For $S=100$, the $L=10$ sinusoidal wall lies in the linearly destabilizing regime (figure 6b) and in figure 15b we see the difference in mean $E$ for different walls. Compared to $L=150$ sinusoidal wall, the solution for $L=10$ wall has higher mean $E$ in the time period shown and finer scales. For a linearly stabilising 
$L=15$ wall for $S=100$ (figure $6 \mathrm{~b}$ ) we observe a lower mean $E$ and quasi-periodic behavior (not shown).

\section{Energy Maps}

A broader view of the combined influence of elasticity and topography on the nonlinear dynamics is obtained by constructing energy maps as shown in figure 16 for $S=100,200$ and 300 for $C_{t}=1$ and $0.4 \leq \mathcal{D} \leq 0.85$. Each point in these plots represents a local extremum of the energy, $E^{*}$, i.e. the value of $E$ at $d E / d t=0$. This corresponds to Poincaré sections in the $E-d E / d T$ energy phase plane [42]. For a particular $\mathcal{D}$, a distinct individual point on these energy maps represents a travelling wave, multiple but countable points represent period- 1 (two points), period-2 (four points) or higher periodic solutions and a dense set of points represents a quasi-periodic or chaotic attractor. The flat wall solutions for $S=300$ in figure 16a has several bifurcations that separate travelling wave, period-1 and period-2 solutions. The overall structure of the map remains largely the same when the flat wall is replaced with a $L=150$ sinusoidal wall (figure 16b), however closer inspection of the $L=150$ results shows that there is a higher density of points in the maps and this corresponds to the introduction of finer temporal scales that were discussed above for $\mathcal{D}=0.55$ and 0.85 . This effect can be seen more clearly in figures $16 \mathrm{e}$ and $16 \mathrm{f}$ where results for the flat wall and $L=150$ sinusoidal wall are plotted together. Reducing the surface tension to $S=200$ and 100 (with the energy maps in figure 16 shifted up by a factor of 10 and 100 respectively) tends to produce higher values of $E^{*}$ and earlier transitions to time-periodic or non-stationary dynamics with chaotic dynamics appearing for the flat wall case. Energy maps for linearly destabilising walls ( $L=15$ for $S=200$ and 300 and $L=10$ for $S=100$ ) are shown in figure 16c, and there is an increase in $E^{*}$ relative to the flat wall while for linearly stabilising walls ( $L=25$ for $S=200$ and 300 and $L=15$ for $S=100$ ) a decrease in $E^{*}$ relative to the flat wall is observed (figure 16d).

As discussed in section 4 , the elastic instability is observed for flat walls when $\mathcal{D}-C_{t} / 3>0$. Varying $C_{t}$, we find that energy maps tend to align for a broad range of parameters when $E^{*}$ is plotted against $\mathcal{D}-C_{t} / 3$ as shown in figure 17 . For $L=150$, the dynamics at larger

$C_{t}$ (shallower inclination) are more chaotic (see panel (b)), whereas for $L=15$ and 25 the 

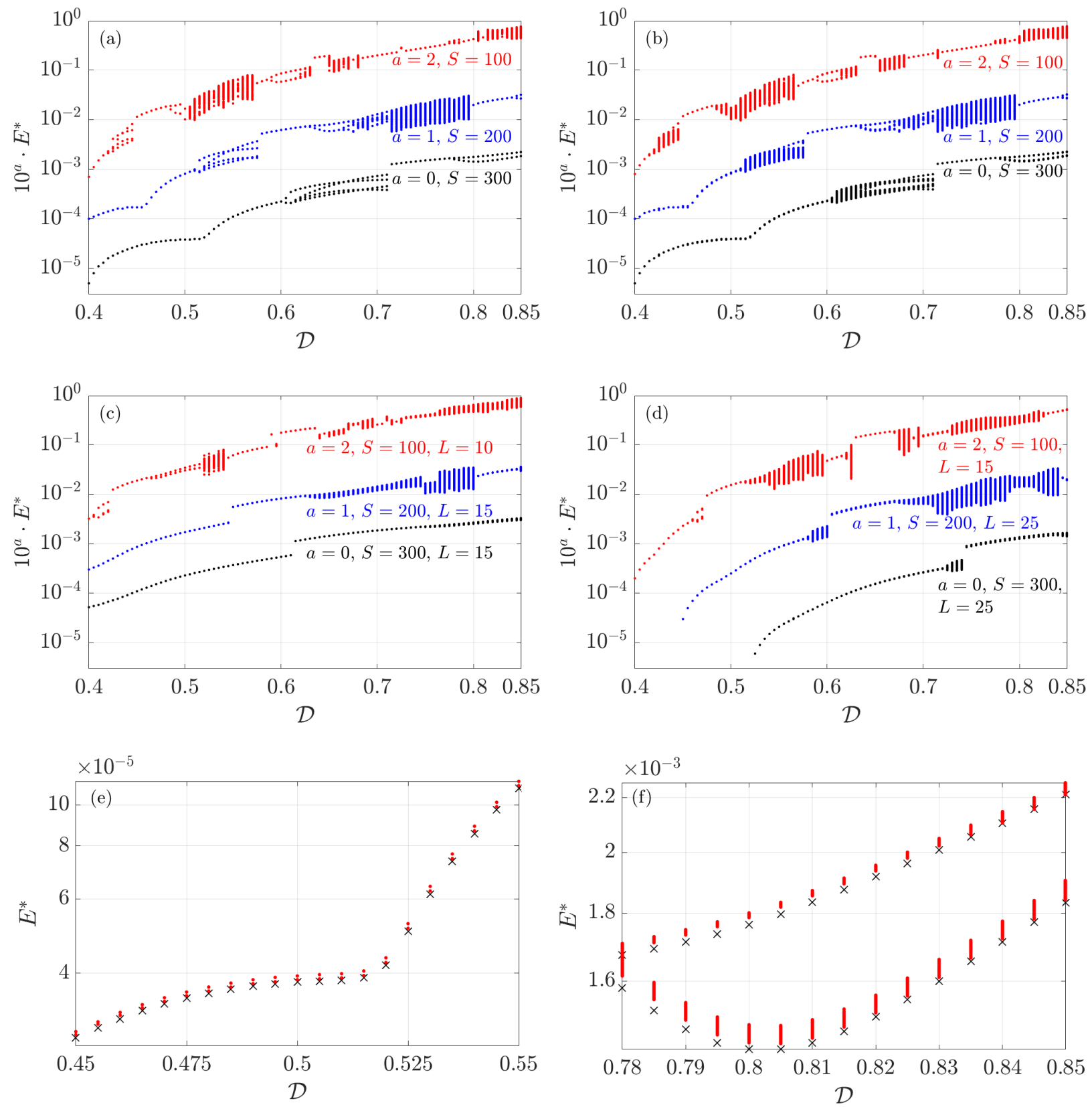

FIG. 16: Maps of the extrema of $E(t)$ with $R e=0, C_{t}=1, L_{D}=150$ and $\delta / L=0.02$ for (a) Flat, (b) $L=150$, (c) destabilising ( $L=15$ for $S=200$ and 300 and $L=10$ for $S=100)$ and (d) stabilising $(L=25$ for $S=200$ and 300 and $L=15$ for $S=100)$ sinusoidal walls at $S=100,200$ and 300 (the first two maps are shifted up by actor of $10^{a}$ with $a$ labeled on each curve ). Superposition of flat (.) and $L=150(\times)$ sinusoidal wall maps with $S=300$ plotted for (e) $0.45 \leq \mathcal{D} \leq 0.55$ and (f) $0.78 \leq \mathcal{D} \leq 0.85$. 
dynamics are very similar for the three inclinations shown (see panels (c) and (d)). Relative to the flat wall, the qualitative effects of $L=150$, destabilising ( $L=15$ ), and stabilising ( $L=25)$ walls are similar for all three values of $C_{t}$ with the introduction of finer scales, an increase in mean $E^{*}$, and a decrease in mean $E^{*}$, respectively.
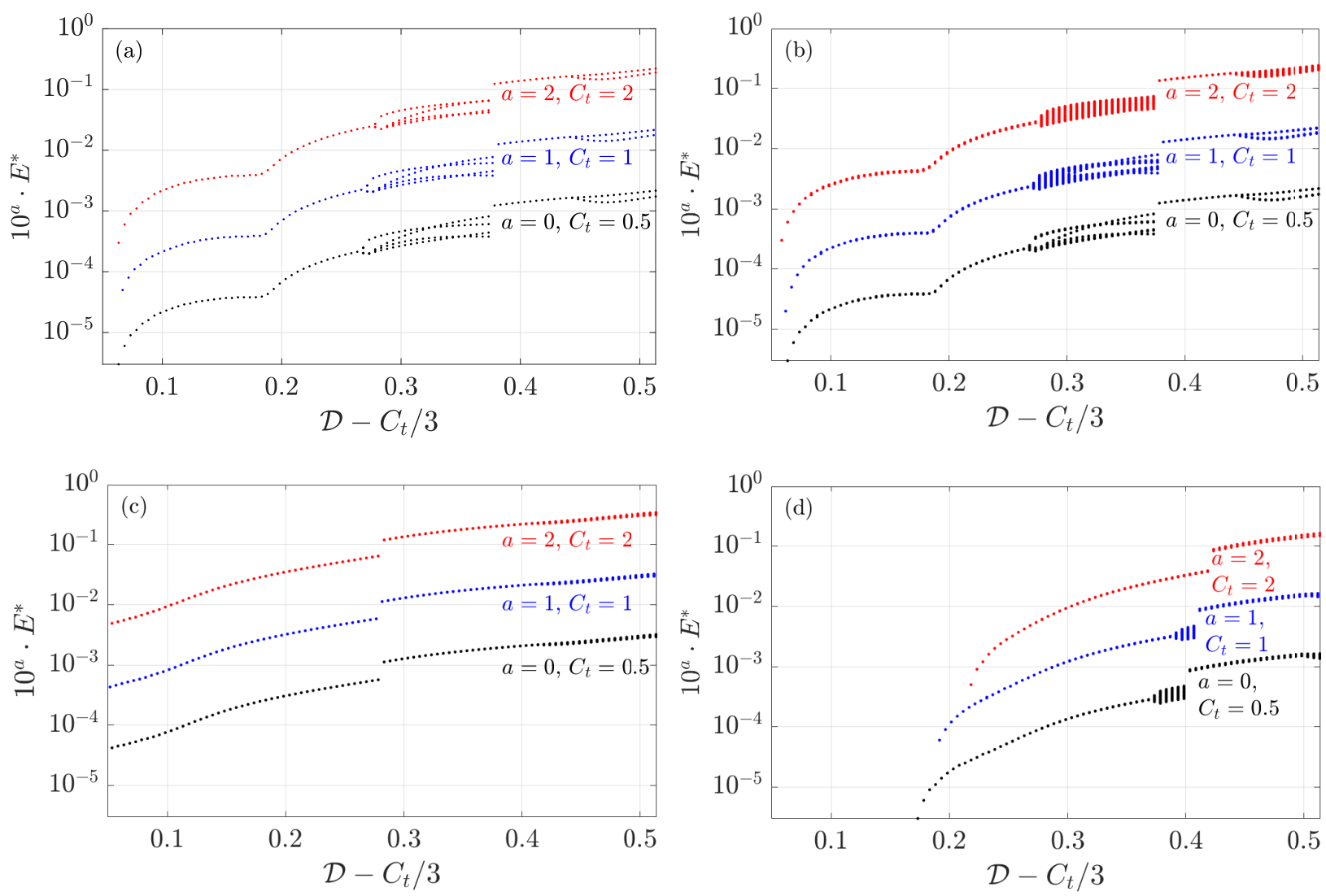

FIG. 17: Maps of the extrema of $E(t)$ with $R e=0, S=300, L_{D}=150$ and $\delta / L=0.02$ for (a) Flat, (b) $L=150$, (c) $L=15$ and (d) $L=25$ sinusoidal walls for $C_{t}=0.5,1$ and 2 (the latter two maps are shifted up by a factor of $10^{a}$ with $a$ labeled on each curve).

\section{Influence of inertia}

We compare finite-Re and inertialess cases using a similar approach to that used when varying $C_{t}$. As noted in section 4 , for flat walls, inertia and elasticity contribute to the linear dynamics in the combination $V / 3=\mathcal{D}+2 / 5 R e$, and we will use this parameter to 'align' the following three cases 1 ) $R e=0, \mathcal{D}$ varying; 2) $\mathcal{D}=0$, Re varying; 3) $\mathcal{D}$ and Re both varying 
with $\mathcal{D}=2 / 5 R e$. We set $S=300, C_{t}=1$ and $L_{D}=150$ and vary the wall wavelength as before. The corresponding energy maps are shown in figure 18. First, we note that the parameter $V$ does tend to align the maps, and clear qualitative similarities are observed for $\mathcal{D} \lesssim 0.6$ for each of the four topographies shown. At larger $\mathcal{D}$, clear differences can be seen. For example, inertia delays the second bifurcation for the flat wall and $L=150$ cases while bringing it forward to smaller values of $V$ when $L=25$. These maps also show that the effect of replacing a flat wall with a $L=150$ sinusoidal wall for the finite-Re cases is very similar to the inertialess-elastic case (figure 18a vs. 18b). The effect of destabilising ( $L=15)$ and stabilising $(L=25)$ walls on the nonlinear dynamics is also similar to the inertialess cases as shown in figures $18 \mathrm{c}$ and $18 \mathrm{~d}$.
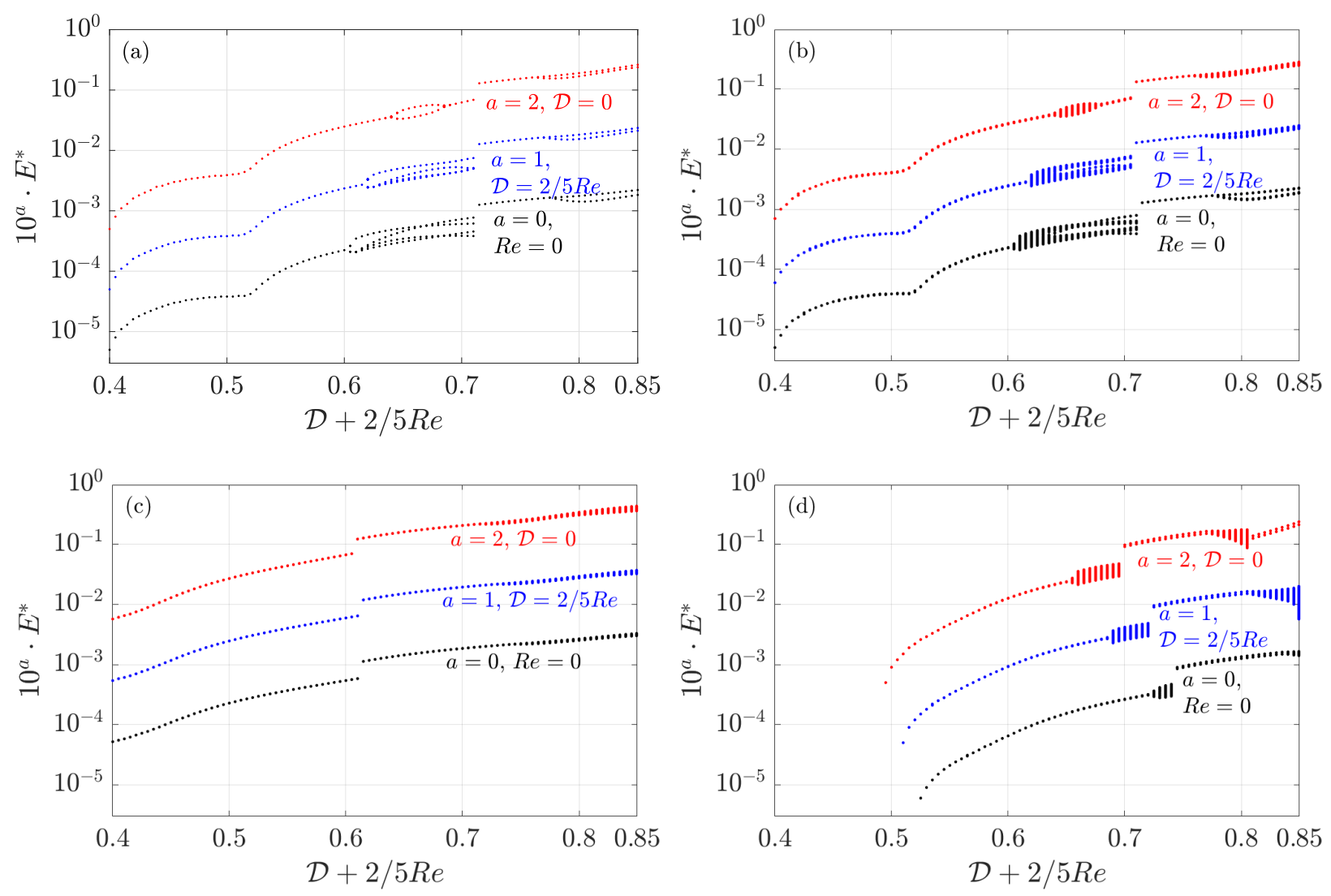

FIG. 18: Maps of the extrema of $E(t)$ with $S=300, C_{t}=1, L_{D}=150$ and $\delta / L=0.02$ for (a) Flat, (b) $L=150$, (c) $L=15$ and (d) $L=25$ sinusoidal walls for $\mathcal{D}=V / 3, V / 6$ and 0 (the remaining contribution to $V / 3=\mathcal{D}+2 / 5 R e$ is from inertia $R e$, and the latter two maps are shifted up by a factor of $10^{a}$ with $a$ labeled on each curve). 


\section{CONCLUSIONS}

The influence of sinusoidal wall topography on the linear stability and nonlinear dynamics of thin gravity-driven viscoelastic films has been analyzed using the modified viscoelastic Benney equation introduced by [1]. Steady-state and linear stability results were organized into three regimes based on the wall wavelength, L. For shorter wall wavelengths, surface tension tends to 'flatten' the steady-state free surface, and linear stability results are similar to those found for flat walls. At intermediate wavelengths, both steady state and linear stability calculations show much greater sensitivity to wall deformation. As $L$ is increased within this regime, topography initially exerts a destabilizing influence, however increasing $L$ further leads to a rapid change and the topography tends to stabilize the flow. At still larger wavelengths, the steady state film thickness tends towards a constant value, and topography ceases to affect the stability results. A decomposition of the growth rate into three components was developed from the linearized equations, and applying this decomposition within the 'intermediate' range of wavelengths showed that the flat-wall instability mechanism was substantially modified by topography. The streamwise velocity perturbation at the free surface (particularly the component normal to the base-state free surface) enhances instability while streamwise variations in the streamwise base-state velocity (coupled with the free-surface perturbation) tend to stabilize the flow. The details of the balance between these effects leads to the initial destabilization followed by stabilization observed within the intermediate range as $L$ is increased. It was also shown that appropriately scaled results for Newtonian films exhibited very similar trends.

Nonlinear simulations generally showed that 'more-unstable' cases (e.g. flows with higher $\mathcal{D}$ or a destabilizing wall wavelength) saturated at higher average values of the energy, E. A broad range of nonlinear states was observed including traveling waves, time-periodic waves, quasi-periodic waves, and chaos. Topography was shown to introduce finer temporal scales and increase the dimension of the underlying attractor. The influences of inclination and inertia were partly explained by relying on linear stability results for flat walls, and increasing surface tension was shown to reduce linear growth rates, shift the range of influential wall wavelengths to larger values of $L$, reduce the average $E$ of saturated nonlinear states, and to produce more regular dynamics. 
We are not aware of experiments which these results can be directly compared to, however Shaqfeh et al.[13] provide a thorough discussion of the dimensional fluid and flow parameters that correspond to viscoelastic films which could be produced in a laboratory setting (and reasonably modeled with the Oldroyd-B equation). This discussion can be applied directly to the present study with the further restriction of a large surface tension. We then expect the results presented here to most readily apply to gravity driven films of dilute polymeric solutions with thicknesses of the order of $10^{-4} \mathrm{~m}$. In many practical cases, the working fluid is either a concentrated solution or a polymer melt and more complicated constitutive models should then be considered. Additionally, non-sinusoidal wall topographies are also of interest, and important steps in these directions have already been taken[e.g. 43-45]. The present study and the trends highlighted here provide a foundation for further progress in the analysis of these more complex flows.

\section{ACKNOWLEDGMENTS}

DTP received support from EPSRC grant EP/L020564/1.

\section{Appendix A: Further details on formulation}

In this section, we provide details on the steps leading from the initial asymptotic expansion (A1) to the final nonlinear evolution equation (5). The dependent variables are expanded as:

$$
\left(u_{i}, p, a_{i j}\right)=\left(u_{i}^{(0)}, p^{(0)}, a_{i j}^{(0)}\right)+\epsilon\left(u_{i}^{(1)}, p^{(1)}, a_{i j}^{(1)}\right)+\epsilon^{2}\left(u_{i}^{(2)}, p^{(2)}, a_{i j}^{(2)}\right)+\mathcal{O}\left(\epsilon^{3}\right),
$$

and these variables are functions of $\tilde{x}_{1}, x_{2}$, and $\tilde{t}$ only. The continuity equation at leading

order gives, $u_{2}^{(0)}=0$, and substituting the expansion into the Oldroyd-B equations (while assuming $D e \sim O(1)$ ), the polymer stresses are found to be,

$$
\begin{gathered}
a_{11}^{(0)}=2 D e\left(\frac{\partial u_{1}^{(0)}}{\partial x_{2}}\right)^{2}, a_{12}^{(0)}=\frac{\partial u_{1}^{(0)}}{\partial x_{2}}, a_{22}^{(0)}=0 \\
a_{12}^{(1)}=\frac{\partial u_{1}^{(1)}}{\partial x_{2}}-D e\left(\frac{\partial^{2} u_{1}^{(0)}}{\partial x_{2} \partial \tilde{t}}+u_{1}^{(0)} \frac{\partial^{2} u_{1}^{(0)}}{\partial x_{2} \partial \tilde{x_{1}}}+u_{2}^{(0)} \frac{\partial^{2} u_{1}^{(0)}}{\partial x_{2}^{2}}+2 \frac{\partial u_{1}^{(0)}}{\partial \tilde{x}_{1}} \frac{\partial u_{1}^{(0)}}{\partial x_{2}}\right) .
\end{gathered}
$$


These results are then substituted into the momentum equations (at the appropriate order) which can then be integrated across the film. At the free surface, the needed boundary conditions are obtained by substituting the expansion (A1) into the full normal stress and tangential stress boundary conditions which are,

$$
\begin{gathered}
p=\frac{1}{1+\left(\partial f / \partial x_{1}\right)^{2}}\left(\beta\left(2 \frac{\partial u_{1}}{\partial x_{1}}\left(\frac{\partial f}{\partial x_{1}}\right)^{2}-\left(\frac{\partial u_{1}}{\partial x_{2}}+\frac{\partial u_{2}}{\partial x_{1}}\right) \frac{\partial f}{\partial x_{1}}+2 \frac{\partial u_{2}}{\partial x_{2}}\right)\right)+ \\
(1-\beta)\left(a_{11}\left(\frac{\partial f}{\partial x_{1}}\right)^{2}-2 a_{12} \frac{\partial f}{\partial x_{1}}+a_{22}\right)-S \frac{\partial^{2} f / \partial x_{1}^{2}}{\left(1+\left(\partial f / \partial x_{1}\right)^{2}\right)^{\frac{3}{2}}} \\
\beta\left(2 \frac{\partial f}{\partial x_{1}}\left(\frac{\partial u_{2}}{\partial x_{2}}-\frac{\partial u_{1}}{\partial x_{1}}\right)+\left(\frac{\partial u_{1}}{\partial x_{2}}+\frac{\partial u_{2}}{\partial x_{1}}\right)\left(1-\left(\frac{\partial f}{\partial x_{1}}\right)^{2}\right)\right)+ \\
(1-\beta)\left(\left(a_{22}-a_{11}\right) \frac{\partial f}{\partial x_{1}}+a_{12}\left(1-\left(\frac{\partial f}{\partial x_{1}}\right)^{2}\right)\right)=0
\end{gathered}
$$

where $f=h+s$. Using the resulting conditions to complete the integration of the momentum equations across the film, we arrive at the needed expressions for the velocity and pressure,

$$
\begin{gathered}
u_{1}^{(0)}=-\frac{3}{2}\left(x_{2}-s\right)^{2}+3\left(x_{2}-s\right) h, \\
u_{1}^{(1)}=9 R e\left[\frac{1}{24}\left[\left(x_{2}-s\right)^{4}-4 h^{3}\left(x_{2}-s\right)\right] h-\frac{1}{6}\left[\left(x_{2}-s\right)^{3}-3 h^{2}\left(x_{2}-s\right)\right] h^{2}\right] \frac{\partial h}{\partial \tilde{x}_{1}}+ \\
\frac{1}{2}\left[3 C_{t} \frac{\partial(h+s)}{\partial \tilde{x}_{1}}-S \epsilon^{2} \frac{\partial^{3}(h+s)}{\partial \tilde{x}_{1}^{3}}-9 \mathcal{D} h \frac{\partial h}{\left.\partial \tilde{x}_{1}\right]}\left(\left(x_{2}-s\right)^{2}-2 h\left(x_{2}-s\right)\right),\right. \\
u_{2}^{(1)}=-\frac{3}{2} \frac{\partial(h+s)}{\partial \tilde{x}_{1}}\left(x_{2}-s\right)^{2}+3 \frac{\partial s}{\partial \tilde{x}_{1}} h\left(x_{2}-s\right), \\
p^{(0)}=-3 C_{t}\left(x_{2}-(h+s)\right)-S \epsilon^{2} \frac{\partial^{2}(h+s)}{\partial \tilde{x}_{1}^{2}} .
\end{gathered}
$$

Note that we have assumed $R e \ll 1 / \epsilon$, so at leading order, inertial effects are neglected leading to a parabolic streamwise velocity profile (A4a). The streamwise velocities from equations (A4a) and (A4b) are used to obtain the volume flow rate,

$$
q\left(x_{1}, t\right)=h^{3}+\frac{6}{5} \operatorname{Reh}^{6} \frac{\partial h}{\partial x_{1}}+3 \mathcal{D} h^{4} \frac{\partial h}{\partial x_{1}}-C_{t} h^{3} \frac{\partial(h+s)}{\partial x_{1}}+\frac{1}{3} S h^{3} \frac{\partial^{3}(h+s)}{\partial x_{1}^{3}},
$$


where we have now moved back to the original streamwise coordinate, $x_{1}$. Substituting equation (A5) into the kinematic condition (3), we obtain the non-linear evolution equation for the film surface (5). This equation retains terms up to $O(\epsilon)$ as is common in studies using the Benney equation and close variants. The stabilizing influence of surface tension enters the equation at this order, and in its absence, discontinuous shock-like solutions are expected to develop[4] for linearly-unstable configurations. The assumption of $S \sim 1 / \epsilon^{2}$ ensures that: 1) a regularizing effect is present, and 2) viscoelastic and inertial effects can be retained for $D e, R e \sim O(1)$. Equivalent assumptions were made by $[1,18]$.

\section{REFERENCES}

[1] Luis Antonio Davalos-Orozco, "Stability of thin viscoelastic films falling down wavy walls," Interfacial Phenomena and Heat Transfer 1 (2013).

[2] Steven J. Weinstein and Kenneth J. Ruschak, "Coating flows," Annual Review of Fluid Mechanics 36, 29-53 (2004).

[3] Chia-Shun Yih, "Stability of Liquid Flow down an Inclined Plane," The Physics of Fluids 6 (1963).

[4] Serafim Kalliadasis, Christian Ruyer-Quil, Benoit Scheid, and Manuel García Velarde, Falling liquid films, Vol. 176 (Springer Science \& Business Media, 2011).

[5] H. Chang, "Wave evolution on a falling film," Annual Review of Fluid Mechanics 26, 103-136 (1994).

[6] R. V. Craster and O. K. Matar, "Dynamics and stability of thin liquid films," Reviews of Modern Physics 81, 1131 (2009).

[7] A T Listrov, "On the stability of the flow of a viscoelastic fluid down an inclined plane," Journal of Applied Mechanics and Technical physics , 67-69 (1965).

[8] A. S. Gupta, "Stability of a visco-elastic liquid film flowing down an inclined plane," Journal of Fluid Mechanics (1967), 10.1017/S0022112067001879.

[9] A. S. Gupta and Lajpat Rai, "Note on the stability of a visco-elastic liquid film flowing down an inclined plane," Journal of Fluid Mechanics 33, 87-91 (1968).

[10] Alexander Morozov and Saverio E. Spagnolie, "Introduction to complex fluids," in Complex Fluids in Biological Systems, edited by S.E. Spagnolie (Springer, New York, 2015) Chap. 1, 
pp. $3-52$.

[11] A. S. Gupta and Lajpat Rai, "Stability of an elastico-viscous liquid film flowing down an inclined plane," Mathematical Proceedings of the Cambridge Philosophical Society 63, 527?536 (1967).

[12] Wei Lai, "Stability of an elastico-viscous film flowing down an inclined plane," The Physics of Fluids 10, 844-847 (1967).

[13] Eric S. G. Shaqfeh, Ronald G. Larson, and Glenn H. Fredrickson, "The stability of gravity driven viscoelastic film-flow at low to moderate reynolds number," Journal of Non-Newtonian Fluid Mechanics 31, 87-113 (1989).

[14] KangPing Chen, "The onset of elastically driven wavy motion in the flow of two viscoelastic liquid films down an inclined plane," Journal of Non-Newtonian Fluid Mechanics 45, 21-45 (1992).

[15] Chao-Tsai Huang and Bamin Khomami, "The instability mechanism of single and multilayer newtonian and viscoelastic flows down an inclined plane," Rheologica Acta 40, 467-484 (2001).

[16] B. S. Dandapat and A. S. Gupta, "Long waves on a layer of a visco-elastic fluid down an inclined plane," Rheologica Acta 17, 492-499 (1978).

[17] S. W. Joo, "The stability and nonlinear flow developments of a viscoelastic draining film with shear thinning," Journal of Non-Newtonian Fluid Mechanics 51, 125-140 (1994).

[18] Feng Kang and Kang Ping Chen, "Nonlinear elastic instability of gravity-driven flow of a thin viscoelastic film down an inclined plane," Journal of Non-Newtonian Fluid Mechanics 57, 243-252 (1995).

[19] Sergey Saprykin, Rudy J. Koopmans, and Serafim Kalliadasis, "Free-surface thin-film flows over topography: influence of inertia and viscoelasticity," Journal of Fluid Mechanics 578, 271-293 (2007).

[20] Alexander Oron and O. Gottlieb, "Nonlinear dynamics of temporally excited falling liquid films," Physics of Fluids (1994-present) 14, 2622-2636 (2002).

[21] Robert Byron Bird, Robert Calvin Armstrong, Ole Hassager, and Charles F. Curtiss, Dynamics of Polymeric Liquids, Vol. 2 (Wiley New York, 1977).

[22] E. S. Shaqfeh, "Fully Elastic Instabilities in Viscometric Flows," Annual Review of Fluid Mechanics 28, 129-185 (1996). 
[23] L. E. Stillwagon and R. G. Larson, "Leveling of thin films over uneven substrates during spin coating," Physics of Fluids A: Fluid Dynamics (1989-1993) 2, 1937-1944 (1990).

[24] A. Wierschem, C. Lepski, and N. Aksel, "Effect of long undulated bottoms on thin gravitydriven films," Acta Mechanica 179, 41-66 (2005).

[25] Alexander Oron and Christian Heining, "Weighted-residual integral boundary-layer model for the nonlinear dynamics of thin liquid films falling on an undulating vertical wall," Physics of Fluids 20 (2008), 10.1063/1.2969410.

[26] Yu Ya Trifonov, "Stability of a viscous liquid film flowing down a periodic surface," International Journal of Multiphase Flow 33, 1186-1204 (2007).

[27] S. J. D. DAlessio, J. P. Pascal, and H. A. Jasmine, "Instability in gravity-driven flow over uneven surfaces," Physics of Fluids 21, 062105 (2009).

[28] L. A. Dávalos-Orozco, "Nonlinear instability of a thin film flowing down a smoothly deformed surface," Physics of Fluids (1994-present) 19, 074103 (2007).

[29] D. Tseluiko, M. G. Blyth, and D. T. Papageorgiou, "Stability of film flow over inclined topography based on a long-wave nonlinear model," Journal of Fluid Mechanics 729, 638-671 (2013).

[30] C. Heining and N. Aksel, "Effects of inertia and surface tension on a power-law fluid flowing down a wavy incline," International Journal of Multiphase Flow 36, 847-857 (2010).

[31] R. Usha and B. Uma, "Long waves on a viscoelastic film flow down a wavy incline," International Journal of Non-Linear Mechanics 39, 1589-1602 (2004).

[32] D. J. Benney, "Long waves on liquid films," Journal of Mathematics and Physics 45, 150-155 (1966).

[33] R. Byron Bird and John M. Wiest, "Constitutive equations for polymeric liquids," Annu. Rev. Fluid Mech 27, 169-93 (1995).

[34] Todd R. Salamon, Robert C. Armstrong, and Robert A. Brown, "Traveling waves on vertical films: Numerical analysis using the finite element method," Physics of Fluids (1994-present) 6, 2202-2220 (1994).

[35] Lawrence F. Shampine and Mark W. Reichelt, "The matlab ode suite," SIAM Journal on Scientific Computing 18, 1-22 (1997). 
[36] C. Pozrikidis, "Effect of surfactants on film flow down a periodic wall," Journal of Fluid Mechanics 496, $105 ? 127$ (2003).

[37] Dmitri Tseluiko, M. G. Blyth, Demetrios T. Papageorgiou, and J.-M. Vanden-Broeck, "Viscous electrified film flow over step topography," SIAM Journal on Applied Mathematics 70, 845-865 (2009).

[38] Marc K. Smith, "The mechanism for the long-wave instability in thin liquid films," Journal of Fluid Mechanics 217, 469-485 (1990).

[39] R. E. Kelly, D. A. Goussis, S. P. Lin, and F. K. Hsu, "The mechanism for surface wave instability in film flow down an inclined plane," Physics of Fluids A: Fluid Dynamics 1, 819$828(1989)$.

[40] Yuri Trifonov, "Nonlinear waves on a liquid film falling down an inclined corrugated surface," Physics of Fluids 29, 054104 (2017).

[41] Daniel Reck and Nuri Aksel, "Experimental study on the evolution of traveling waves over an undulated incline," Physics of Fluids 25, 024103 (2013).

[42] Prasun K. Ray, Jordan C. Hauge, and Demetrios T. Papageorgiou, "Nonlinear interfacial instability in two-fluid viscoelastic couette flow," Journal of Non-Newtonian Fluid Mechanics 251, 17-27 (2018).

[43] M. Pavlidis, Y. Dimakopoulos, and J. Tsamopoulos, "Steady viscoelastic film flow over 2d topography: I. the effect of viscoelastic properties under creeping flow," Journal of NonNewtonian Fluid Mechanics 165, 576-591 (2010).

[44] M. Pavlidis, G. Karapetsas, Y. Dimakopoulos, and J. Tsamopoulos, "Steady viscoelastic film flow over 2d topography: II. the effect of capillarity, inertia and substrate geometry," Journal of Non-Newtonian Fluid Mechanics 234, 201-214 (2016).

[45] M. Pradas, D. Tseluiko, C. Ruyer-Quil, and S. Kalliadasis, "Pulse dynamics in a power-law falling film," Journal of Fluid Mechanics 747, 460-480 (2014). 